\title{
REVIEW
}

\section{DISCONNECTED AGING: CEREBRAL WHITE MATTER INTEGRITY AND AGE-RELATED DIFFERENCES IN COGNITION}

\author{
I. J. BENNETT ${ }^{\mathrm{a}}$ AND D. J. MADDEN ${ }^{\mathrm{b}, \mathrm{c} *}$ \\ ${ }^{a}$ Center for the Neurobiology of Learning and Memory, University \\ of California, Irvine, United States \\ ${ }^{\mathrm{b}}$ Brain Imaging and Analysis Center, Duke University Medical \\ Center, United States \\ ${ }^{\mathrm{c}}$ Department of Psychiatry and Behavioral Sciences, Duke University \\ Medical Center, United States
}

\begin{abstract}
Cognition arises as a result of coordinated processing among distributed brain regions and disruptions to communication within these neural networks can result in cognitive dysfunction. Cortical disconnection may thus contribute to the declines in some aspects of cognitive functioning observed in healthy aging. Diffusion tensor imaging (DTI) is ideally suited for the study of cortical disconnection as it provides indices of structural integrity within interconnected neural networks. The current review summarizes results of previous DTI aging research with the aim of identifying consistent patterns of age-related differences in white matter integrity, and of relationships between measures of white matter integrity and behavioral performance as a function of adult age. We outline a number of future directions that will broaden our current understanding of these brain-behavior relationships in aging. Specifically, future research should aim to (1) investigate multiple models of age-brain-behavior relationships; (2) determine the tract-specificity versus global effect of aging on white matter integrity; (3) assess the relative contribution of normal variation in white matter integrity versus white matter lesions to age-related differences in cognition; (4) improve the definition of specific aspects of cognitive functioning related to age-related differences in white matter integrity using information processing tasks; and (5) combine multiple imaging modalities (e.g., resting-state and task-related functional magnetic resonance imaging; fMRI) with DTI to clarify the role of cerebral white matter integrity in cognitive aging.

This article is part of a Special Issue entitled: The CNS White Matter. (C) 2013 IBRO. Published by Elsevier Ltd. All rights reserved.
\end{abstract}

\footnotetext{
${ }^{*}$ Correspondence to: D. J. Madden, Brain Imaging and Analysis Center, Box 2737, Hock Plaza, Duke University Medical Center, Durham, NC 27710, United States. Tel: +1-919-681-9345; fax: + 1-919-681-7033.

E-mail address: david.madden@duke.edu (D. J. Madden).

Abbreviations: AD, axial diffusivity; DTI, diffusion tensor imaging; FA, fractional anisotropy; FLAIR, fluid-attenuated inversion recovery; fMRI, functional MRI; HARDI, high angular resolution diffusion imaging; MD, mean diffusivity; $\mathrm{PCA}$, principal component analysis; $\mathrm{RD}$, radial diffusivity; WML, white matter lesions.
}

Key words: white matter integrity, diffusion tensor imaging, aging, cognition, magnetic resonance imaging, disconnection.

\begin{tabular}{lr}
\multicolumn{1}{c}{ Contents } & 187 \\
Introduction & 188 \\
White matter integrity declines in aging & 192 \\
White matter integrity correlates with cognition in aging & 192 \\
Positive brain-behavior relationships & 192 \\
Age mediates brain-behavior relationships & 194 \\
White matter integrity mediates age-behavior relationships & 195 \\
Considerations for future research & 195 \\
Multiple age-brain-behavior models & 196 \\
Global versus tract-specific age-related effects & 197 \\
WML & 199 \\
General versus task-specific components of performance & 199 \\
Multimodal imaging & 200 \\
Conclusions & 201 \\
Acknowledgements & 201 \\
References &
\end{tabular}

\section{INTRODUCTION}

The broad array of human information processing abilities that we categorize as perception, attention, and memory are the result of coordinated processing among distributed brain regions (Mesulam, 1990; Mclntosh, 2000). Disruptions to communication within these neurocognitive networks lead to cognitive dysfunction recognized as neurological disease (Geschwind, 1965a,b; Catani and Ffytche, 2005; Filley, 2005). But disconnection may also lead to measurable variations in performance that are well below the threshold for neurological disease.

In healthy aging, for example, behavioral research has long established that age-related declines occur in fluid (speed-based) measures, whereas crystallized (knowledge-based) measures are relatively preserved (Craik and Salthouse, 2008). More recently, neuroimaging research has revealed corresponding changes in the aging brain, including decreases in the integrity of the white matter that can occur in the absence of specific neurological disease. These recent findings have created a central role for the concept of cortical disconnection, via age-related declines in white matter integrity, in the cognitive neuroscience of aging 
(O'Sullivan et al., 2001; Bartzokis, 2004; Charlton et al., 2006; Andrews-Hanna et al., 2007; Salat, 2011).

Diffusion tensor imaging (DTI) is ideally suited for the study of cortical disconnection as it provides indices of structural integrity within interconnected neural networks. DTI measures the diffusion, or movement, of molecular water (Basser et al., 1994; Pierpaoli and Basser, 1996; Johansen-Berg and Behrens, 2009). Within fluid-filled spaces of the brain (e.g., ventricles), diffusion is nearly unbounded and thus non-directional (i.e., isotropic). Similarly, within the gray matter, diffusion is relatively isotropic as a result of non-uniform restriction of molecular movement by microstructures such as cell bodies and dendrites. In contrast, diffusion within the white matter is more directional (i.e., anisotropic). In the white matter, microstructures such as axonal cell membranes, myelin sheaths, and neurofilaments restrict molecular movement such that the primary direction of diffusion runs parallel to axons (Pierpaoli et al., 1996; Beaulieu, 2002; Le Bihan, 2003).

Integrity of white matter structures can be inferred from DTI-based measures of the rate and directionality of associated molecular diffusion. For example, fractional anisotropy (FA) is a scalar measure that indexes the anisotropic, or restricted, fraction of total diffusion. Higher FA values (i.e., closer to 1) reflect increased diffusion directionality, independent of the rate of diffusion, which is characteristic of diffusion along the length of white matter axons. Mean diffusivity (MD) indexes the average rate of diffusion, independent of the directionality. Lower MD values within the white matter are characteristic of regions where neural microstructures (e.g., axonal cell membranes, myelin sheaths, and neurofilaments) displace intra- and extra-cellular water.

White matter integrity can be further characterized by measuring the rate of diffusion along the primary (axial diffusivity, AD) and secondary (radial diffusivity, RD) axes of diffusion ellipsoids that summarize diffusion properties of each voxel. Animal studies have provided support for the notion that $A D$ is sensitive to axonal differences and $\mathrm{RD}$ is sensitive to myelin changes (Song et al., 2003, 2005; Sun et al., 2006, 2008). In turn, these findings have guided interpretations of similar patterns in human studies (Bennett et al., 2010; Burzynska et al., 2010). For example, age-related damage to both axon fibers and the surrounding myelin sheaths is suspected when older adults show increases in FA that are accompanied by increases in both RD and $A D$. In contrast, more specific disruption of myelin is inferred when age-related RD increases occur in the absence of $A D$ increase. These interpretations of the neurobiological substrates of $A D$ and $R D$ are limited when not considered in the context of the orientation of the principal diffusion direction (Wheeler-Kingshott and Cercignani, 2009). However, the utility of diffusivity measures (MD, $A D$, and $R D$ ) is strengthened by the notion that they may be more sensitive to white matter integrity differences than their more commonly used counterpart, FA. That is, in some cases, complementary changes in both $A D$ and $R D$ may lead to $F A$ being relatively unchanged.
Beyond the measurement of white matter properties, DTI provides a basis for the anatomical reconstruction of white matter tracts throughout the brain. An illustration of these major white matter pathways is presented in Fig. 1. DTI tractography (or fiber tracking) algorithms estimate connections among interconnected voxels by propagating from a specified source region or between source and target regions. This reconstruction may be conducted in the native anatomical space of each participant, or in an averaged space that normalizes the anatomy across individual participants. Tractography and other voxel-wise analyses of DTI data have further variants, each with advantages and disadvantages, though a comparison of these different methodologies is beyond the scope of this article (Mori and van Zijl, 2002; Wakana et al., 2004; Catani and Ffytche, 2005; Catani, 2006; Smith et al., 2006; Nucifora et al., 2007; Jones, 2008). Tractography is emerging as an informative method for characterizing the major pathways of the white matter in the brain. It is important to note, however, that tractography and related DTI indices are measures of water diffusion and thus are indirect measures of structural connectivity (Jones et al., 2013). Further, structural and functional connectivity are not identical, because strong functional connections may exist between regions with limited structural connections (Honey et al., 2009).

Researchers frequently use DTI to study age-related differences in cerebral white matter integrity, as well as the relationships between measures of white matter integrity and behavioral performance as a function of adult age. The technology of DTI and related techniques, such as q-ball imaging and high angular resolution diffusion imaging (HARDI) continues to evolve, providing improved resolution of the orientation of white matter pathways (Tuch et al., 2003; Koenig et al., 2013; Nagy et al., 2013; Zhan et al., 2013). The majority of the research to date, however, addressing the relation of white matter integrity to age-related differences in cognition, has used conventional DTI and fiber tractography. The current review aims to summarize consistent patterns of results across these studies, discuss interpretations that can be drawn from DTI studies of cognitive aging and their limitations, and outline future directions that will broaden our current understanding of these brain-behavior relationships in aging.

\section{WHITE MATTER INTEGRITY DECLINES IN AGING}

Age-related declines in cerebral white matter integrity are well-documented, and the number of DTI investigations addressing age-related integrity differences is growing rapidly (Sullivan and Pfefferbaum, 2006, 2007; Wozniak and Lim, 2006; Malloy et al., 2007; Minati et al., 2007; Madden et al., 2009a, 2012; Salat, 2011; Carmichael and Lockhart, 2012). Across studies, the predominant findings are decreased $F A$ and increased $M D$ as a function of increasing adult age, suggesting an agerelated decline in the composition and integrity of the 


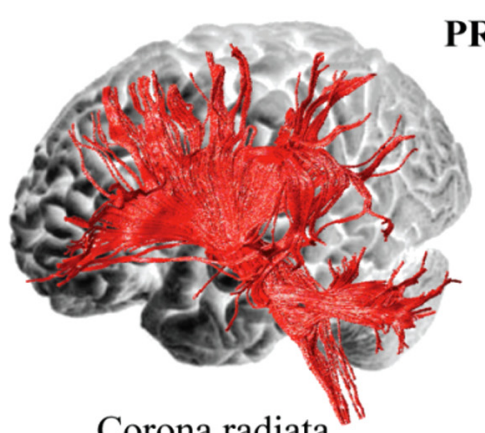

PROJECTION

Corona radiata

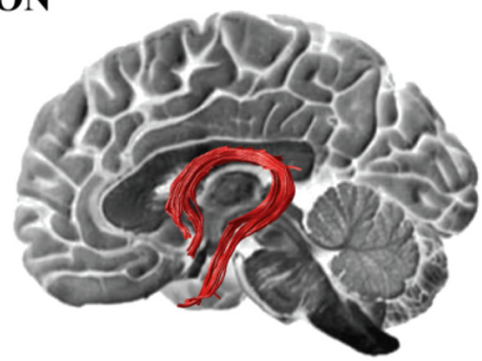

Fornix

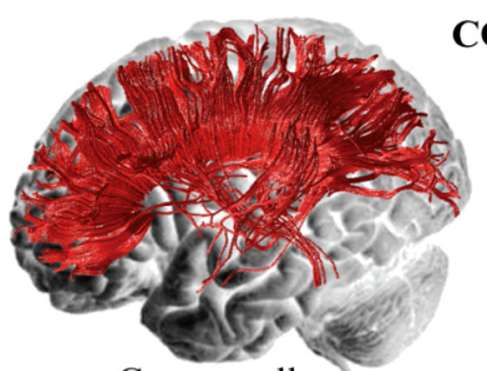

Corpus callosum

COMMISSURAL

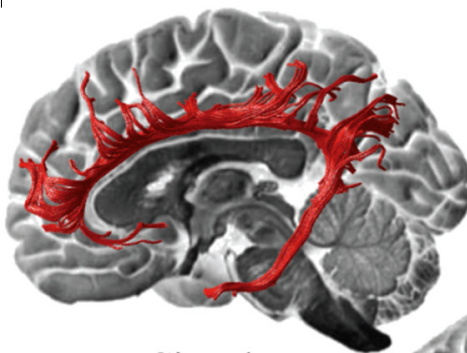

Cingulum
ASSOCIATION

Anterior commissure

Anterior commissure

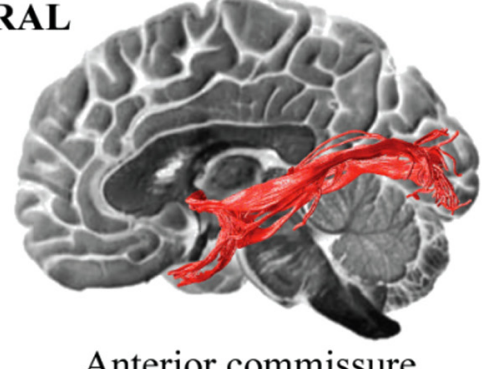

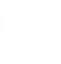

$$
\text { . }
$$




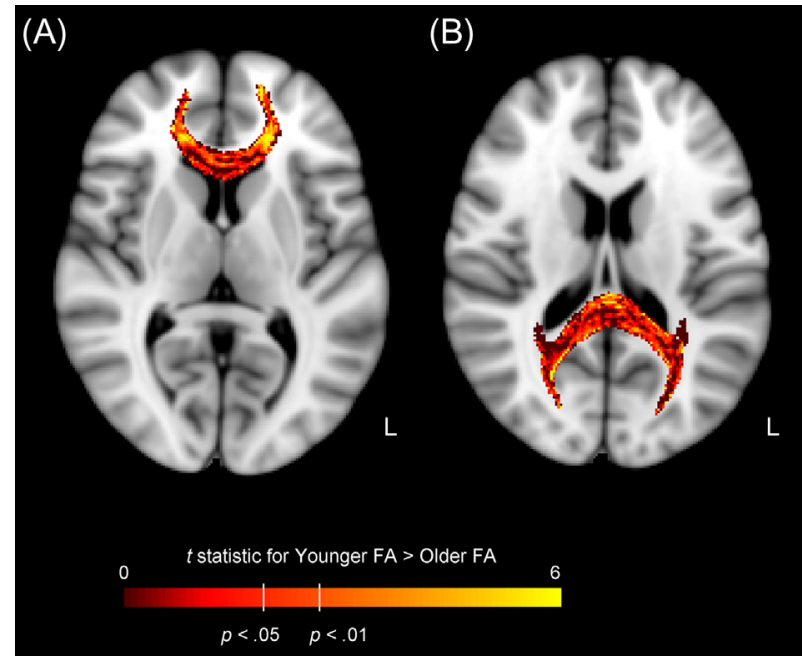

Fig. 2. Difference in fractional anisotropy (FA) from diffusion tensor imaging, for the genu (Panel A) and splenium (Panel B) of the corpus callosum, for younger and older adults. Data are voxelwise $t$ maps of the younger $>$ older FA difference score, across 23 younger adults (19-39 years of age) and 23 older adults (60-79 years of age). Participants were healthy, community-dwelling individuals without any sign of cognitive impairment on neuropsychological testing or history of cardiovascular disease (other than hypertension). Data are overlaid on a T1-weighted template, in radiological orientation (left = right). Age-related decline in FA is most prominent in the lateral segments of the genu. Authors' unpublished data.

white matter. The age-related decrease in FA is illustrated in Fig. 2. These age-related differences in white matter integrity are accompanied by substantial variability across individuals, and the ranges of DTI measures overlap across adult age groups. Nonetheless, the pattern of age-related white matter integrity decline observed across cross-sectional studies is further supported by longitudinal studies, which have shown similar white matter integrity changes (i.e., age-related decrease in FA and increase in MD) in as little as one year within healthy older populations (Barrick et al., 2010; Teipel et al., 2010; cf. Sullivan et al., 2010b).

Across studies, age group differences in white matter integrity are also more prominent for RD than $A D$ (Bhagat and Beaulieu, 2004; Davis et al., 2009; Madden et al., 2009b; Zhang et al., 2010; Bennett et al., 2010; Burzynska et al., 2010). In addition, RD is typically higher for older adults relative to younger adults, whereas both age-related increases and decreases in $A D$ have been reported (Zahr et al., 2009; Bennett et al., 2010; Sullivan et al., 2010b; Burgmans et al., 2011). This pattern of results is illustrated in Fig. 3, in which older adults show increased RD in the genu of the corpus callosum and superior longitudinal fasciculus relative to younger adults, whereas there are no significant age group differences in $A D$ across tracts. These age-related differences in white matter integrity are consistent with known changes in the aging brain. For example, post-mortem histological studies have shown that healthy aging is accompanied by axonal and myelin degeneration and deformation (e.g., swelling, redundant wrapping), in addition to other changes to the cellular environment (e.g., accumulation of cellular debris, formation of glial scars) (Meier-Ruge et al., 1992; Aboitiz et al., 1996; Tang et al., 1997; Peters, 2002; Marner et al., 2003).

Another measure of white matter integrity gaining attention in the DTI literature is mode (MO; Ennis and Kindlmann, 2006), which indexes the shape of anisotropy as a continuous measure. As illustrated in Fig. 4, whereas FA indicates the degree of restricted diffusion, MO can reveal whether diffusion is primarily restriction in a single direction (resulting in a planar, pancake-shaped ellipsoid) or in two directions (resulting in a linear, cigar-shaped ellipsoid). Higher $\mathrm{MO}$ values (approaching 1) reflect a more linear diffusion ellipsoid, as is seen in white matter with predominantly one-fiber orientation. Lower MO values (approaching -1) indicate a planar ellipsoid more common to regions with multiple fiber populations (e.g., crossing or kissing fibers). Examining $\mathrm{MO}$ in conjunction with other integrity measures may clarify the neurobiology underlying differences in white matter integrity (Gouttard et al., 2009; Douaud et al., 2011). For example, decreased FA with increased $\mathrm{MO}$ may indicate reduced integrity within a white matter tract, whereas decreased FA with decreased MO may suggest macrostructural changes (e.g., fiber organization) in regions with crossing fibers.

Previous DTI studies suggest that aging has regionspecific effects on white matter integrity. Initial studies
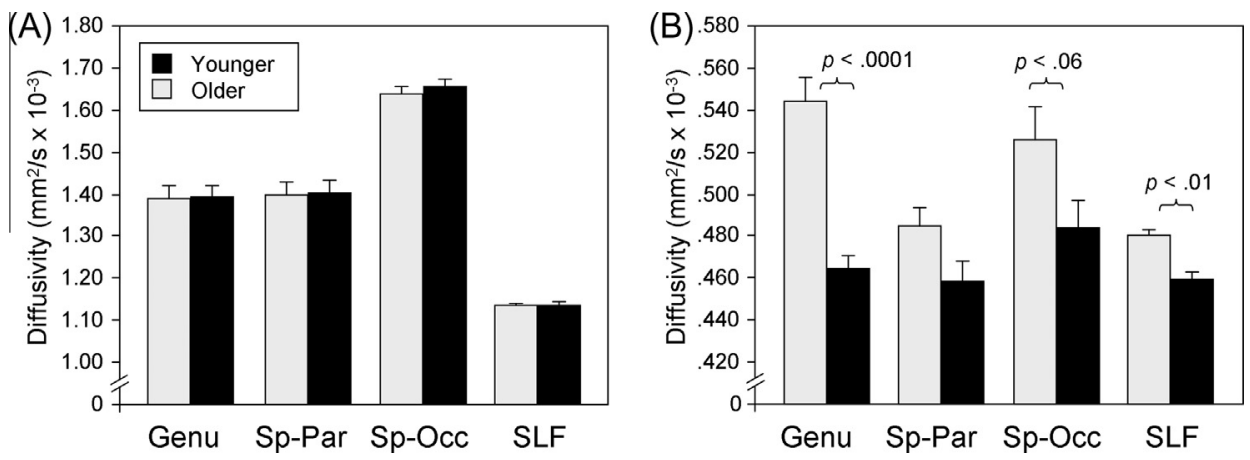

Fig. 3. Age-related differences in axial (Panel A) and radial (Panel B) diffusivity as a function of age group and region. Panel $A$ reveals similar $A D$ between younger (black bars) and older (gray bars) adults across all white matter regions, whereas Panel $B$ reveals increased RD in older adults relative to younger adults in the genu of the corpus callosum, splenium-occipital region (Sp-Occ), and superior longitudinal fasciculus (SLF). SpPar $=$ splenium-parietal. Reproduced with permission from Madden et al. (2009b). 


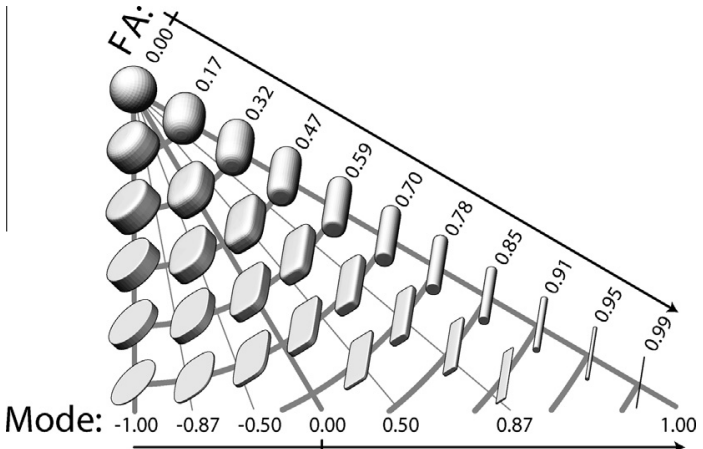

Fig. 4. Demonstration of the space of anisotropy decomposed into two orthogonal channels: fractional anisotropy $\left(\mathrm{FA}=R_{2}\right)$ and mode $\left(=R_{3}=K_{3}\right)$. Each glyph represents the shape of diffusion tensors with constant tensor norm rendered with superquadric glyphs. Increasing distance from the top left spherical glyph indicates increasing FA, whereas the angular deviation from the left edge indicates increasing mode as it transitions from planar anisotropic (mode $=-1)$, to orthotropic (mode $=0$ ), to linear anisotropic (mode $=1$ ). Glyphs along constant radii (constrained to an arc) are of constant fractional anisotropy, but of varying mode. This figure explicitly demonstrates that increases in FA do not necessarily indicate increasing linear anisotropy. The space of FA and mode is correctly diagrammed as an isosceles triangle; note that isocontours of FA are orthogonal to isocontours of mode. Reproduced with permission from Ennis and Kindlmann (2006).

consistently reported larger age-related FA decreases in frontal regions including the genu of the corpus callosum and pericallosal white matter, relative to posterior brain regions (Pfefferbaum et al., 2000; Sullivan et al., 2001; Sullivan and Pfefferbaum, 2003). Results of later studies largely replicated these patterns (Madden et al., 2004, 2007, 2009a; Salat et al., 2005; Bucur et al., 2008; Bennett et al., 2010; Burzynska et al., 2010; Michielse et al., 2010). However, this anterior-posterior gradient does not necessarily represent age-related effects specific to the frontal lobe. Davis et al. (2009) reported that white matter tracts traversing frontal cortex (e.g., uncinate fasciculus and cingulum) exhibited a monotonic decrease in the magnitude of the age-related effect in FA, and that this pattern did not change abruptly at the boundary of the frontal lobe. Further, some posterior white matter regions exhibit larger age-related declines in FA compared to frontal regions (Salat et al., 2005; Bennett et al., 2010). For example, Bennett et al. observed a significant positive relationship between the percent age group difference in FA and the location of white matter clusters, with larger age-related decreases in FA in more anterior clusters. Yet, this anterior-posterior gradient, shown in Fig. 5, was accompanied by significant variability. Most notably, two posterior clusters (left inferior sagittal stratum, right cerebellum) exhibited the largest age group differences in FA. In addition, other researchers have suggested a superiorinferior gradient in which superior white matter is more susceptible to age-related declines relative to inferior regions (Zahr et al., 2009; Sullivan et al., 2010a,b). Thus, the anterior-posterior gradient, considered in isolation, may be an oversimplification of age-related effects in white matter integrity.

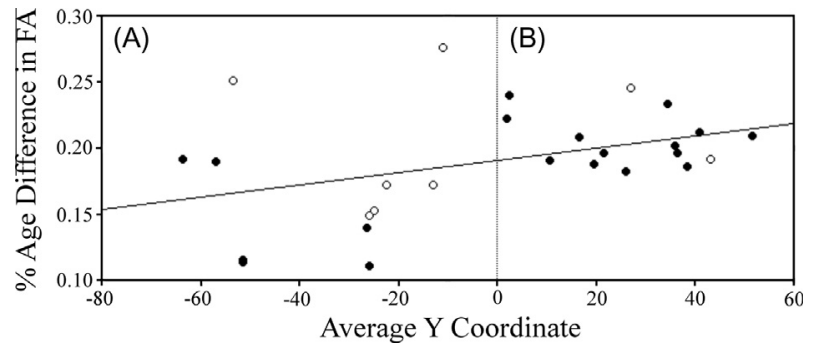

Fig. 5. An anterior-posterior gradient of age-related difference in FA. A significant positive relationship was observed between the percent age group difference in FA ( $y$-axis, calculated for each white matter cluster as FA in older adults minus FA in younger adults, divided by the average FA value for that cluster) and the location of white matter clusters ( $x$-axis, measured as the average y coordinate for each white matter cluster in $\mathrm{mm}$ according to $\mathrm{MNI}$ space). As the regression line indicates, age-related decreases in FA were significantly larger in anterior (Panel B) versus posterior (Panel A) clusters. This relationship was significant for the frequently reported superior clusters (closed circle) with $z$ coordinates above or traversing zero, but did not attain significance for inferior clusters (open circles). However, this anterior-posterior gradient was accompanied by significant variability, with two posterior clusters (left inferior sagittal stratum, right cerebellum) exhibiting the largest age group differences in FA. See original text for white matter clusters that correspond to each $y$ coordinate. Reproduced with permission from Bennett et al. (2010).

Together, the various patterns of age-related differences in white matter integrity are generally consistent with the white matter retrogenesis hypothesis. Also known as last-in-first-out hypothesis, this theory states that white matter regions that myelinate later during development exhibit deterioration earlier during aging (Bartzokis, 2004; Brickman et al., 2012). However, in the same way that some posterior white matter regions do not conform with the anteriorposterior gradient, not all age-related integrity effects accommodate this retrogenesis hypothesis (Westlye et al., 2010). Ultimately, it may be more accurate to categorize the age-related differences in white matter integrity in terms of specific fiber systems (e.g., frontoparietal) rather than in terms of gradients of change (Salat et al., 2005; Salat, 2011). Toward this approach, at least one study separated white matter tracts into functional categories, finding that age had the largest negative effect on association fibers that comprise cortical-cortical connections (e.g., superior longitudinal fasciculus), relative to projection fibers that connect cortical and subcortical regions (e.g., corticospinal tract) and callosal fibers that connect the left and right hemispheres of the brain (Stadlbauer et al., 2008).

In summary, two main conclusions can be drawn from the existing DTI aging literature. First, cerebral white matter integrity typically declines in healthy aging, as seen by age-related decreases in FA and increases in MD. Second, these age effects are likely driven by changes in underlying myelin, as seen by preferential age-related increases in $\mathrm{RD}$ relative to $A D$. Additional research will be necessary to clarify the regionspecificity of these age-related declines in cerebral white matter integrity. 


\section{WHITE MATTER INTEGRITY CORRELATES WITH COGNITION IN AGING}

Of specific interest in the current review is whether agerelated declines in cerebral white matter integrity contribute to cognitive deficits associated with aging, as predicted by cortical disconnection theories (O'Sullivan et al., 2001; Bartzokis, 2004; Charlton et al., 2006; Andrews-Hanna et al., 2007; Salat, 2011). Salthouse (2011) recently concluded that the evidence in support of measures of brain structure as a neuroanatomical substrate of age-related cognitive decline is weak. This limitation can be attributed to the use of inappropriate statistical approaches for interrogating relationships among age, cognitive performance, and white matter integrity. For example, disconnection theories predict age-related declines in both white matter integrity (as described in the previous section) and cognitive performance, and relationships between white matter integrity and cognitive performance. However, separate pair-wise correlations among these variables do not address whether the age-related differences in white matter integrity have a causal influence on the agerelated differences in cognitive performance. Instead, mediation analyses are required to infer a direct or causal role for white matter integrity on cognitive deficits in aging (Baron and Kenny, 1986; Salthouse, 1992; Hayes, 2009; Charlton et al., 2010; Fjell and Walhovd, 2010). When using mediation analyses, white matter integrity can be identified as a significant mediator if integrity is related to cognitive performance independently of age, and the age-related variance in cognitive performance is attenuated when integrity is included in the regression model that predicts cognitive performance using age.

In line with the broader aim of evaluating evidence for cortical disconnection as an explanation for cognitive aging, we previously surveyed the DTI aging literature in two complementary reviews (Madden et al., 2009a, 2012). Across a number of studies, significant relationships were reported between integrity from distributed white matter regions and performance in multiple cognitive domains. Severable notable patterns emerged: (1) relationships between white matter integrity and cognitive performance were predominantly positive, such that increasing integrity was correlated with better performance; (2) the strength of these brainbehavior relationships was weaker when analyses statistically controlled for chronological age; and (3) the strength of age-behavior relationships was weaker when analyses statistically controlled for white matter integrity. Each of these three patterns will be briefly discussed in turn. We have emphasized results from more recent studies that were not featured in our previous reviews in order to identify remaining gaps in the current literature.

\section{Positive brain-behavior relationships}

Consistent with the findings reported in our previous DTI reviews of cognitive aging (Madden et al., 2009a, 2012), recent DTI studies have also observed that increased white matter integrity (i.e., increased FA, decreased diffusivity) relates to better cognitive performance across groups of younger and older adults (Schulze et al., 2011; Coxon et al., 2012), within groups of older adults (Charlton et al., 2009; Sexton et al., 2010; Yassa et al., 2010; Penke et al., 2010a; Forstmann et al., 2011; Metzler-Baddeley et al., 2011; Ystad et al., 2011; Antonenko et al., 2012; Bosch et al., 2012; Brickman et al., 2012; He et al., 2012; Lockhart et al., 2012; Van Impe et al., 2012; Borghesani et al., 2013; Jacobs et al., 2013), and in groups that span childhood to later adulthood (Bendlin et al., 2010; Stamatakis et al., 2011; Salami et al., 2012; Samanez-Larkin et al., 2012; Sasson et al., 2012, 2013).

We previously reported that these positive brainbehavior relationships were strongest for measures of fluid cognition. Consistent with this finding, the majority of recent DTI studies reported that effect sizes for relationships between white matter integrity and cognitive performance were larger for executive function and processing speed (Bendlin et al., 2010; Ystad et al., 2011; Brickman et al., 2012; Salami et al., 2012; Borghesani et al., 2013; Haasz et al., 2013). For example, Ystad et al. (2011) found significant positive relationships between performance on tasks that tap executive processes and FA from three tracts connecting subcortical regions (thalamus, putamen) to frontal regions (inferior, dorsomedial), and between processing speed and FA from separate tracts connecting the putamen to visual and sensorimotor regions. These data are presented in Fig. 6. The only study that reported similarly large effect sizes for memory performance included both healthy older adults and individuals diagnosed with mild cognitive impairment and Alzheimer's Disease in the analyses (He et al., 2012), suggesting that dementia-related changes in white matter integrity may have driven their effects for memory. Nonetheless, these data are consistent with the notion that (age-related) decreases in cerebral white matter integrity are associated with impaired cognitive functioning in older adults.

We further previously reported that, across studies, there was minimal overlap in the region-specificity of these positive brain-behavior relationships, with the exception that the relationships were stronger in frontal brain regions (Madden et al., 2012). Within studies, however, reliable relationships between cognitive performance and integrity from task-relevant regions were observed, as described here for Ystad et al. (2011). These findings suggest that cognitive processes, such as those featured in previous DTI aging studies are complex and rely on multiple brain regions. An important direction for future research will be to isolate component processes of these complex cognitive functions and assess their relation to integrity from specific white matter fiber systems (see the section of this article, General versus Task-Specific Components of Performance, below).

\section{Age mediates brain-behavior relationships}

In addition to finding that increased white matter integrity related to better cognitive performance, our previous DTI 

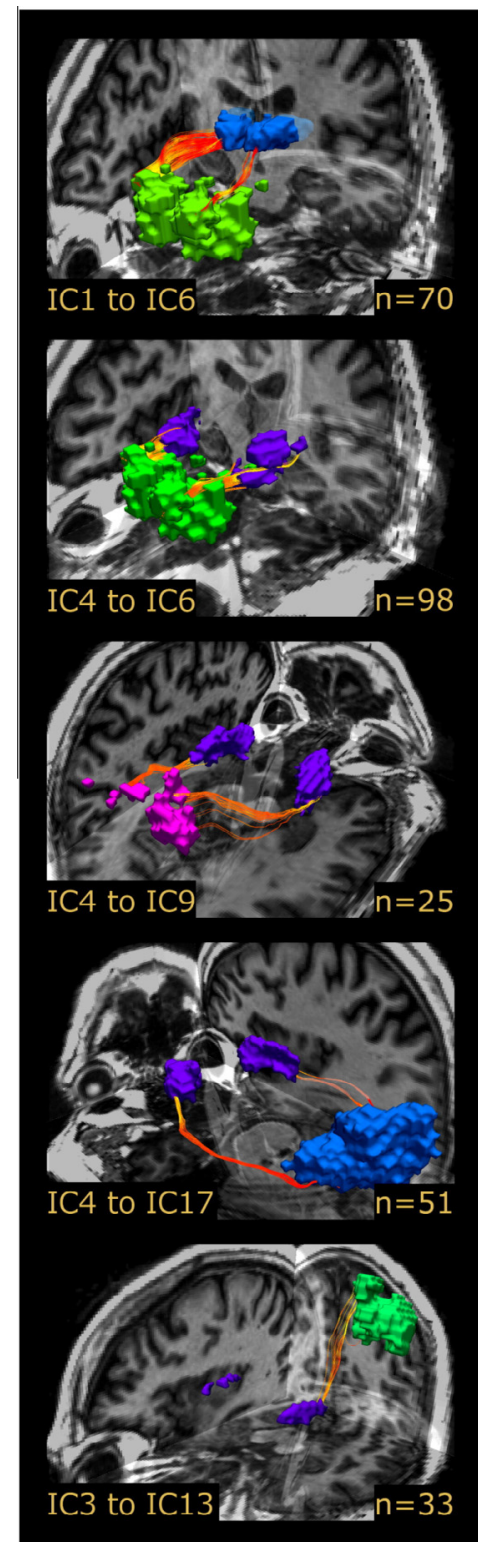
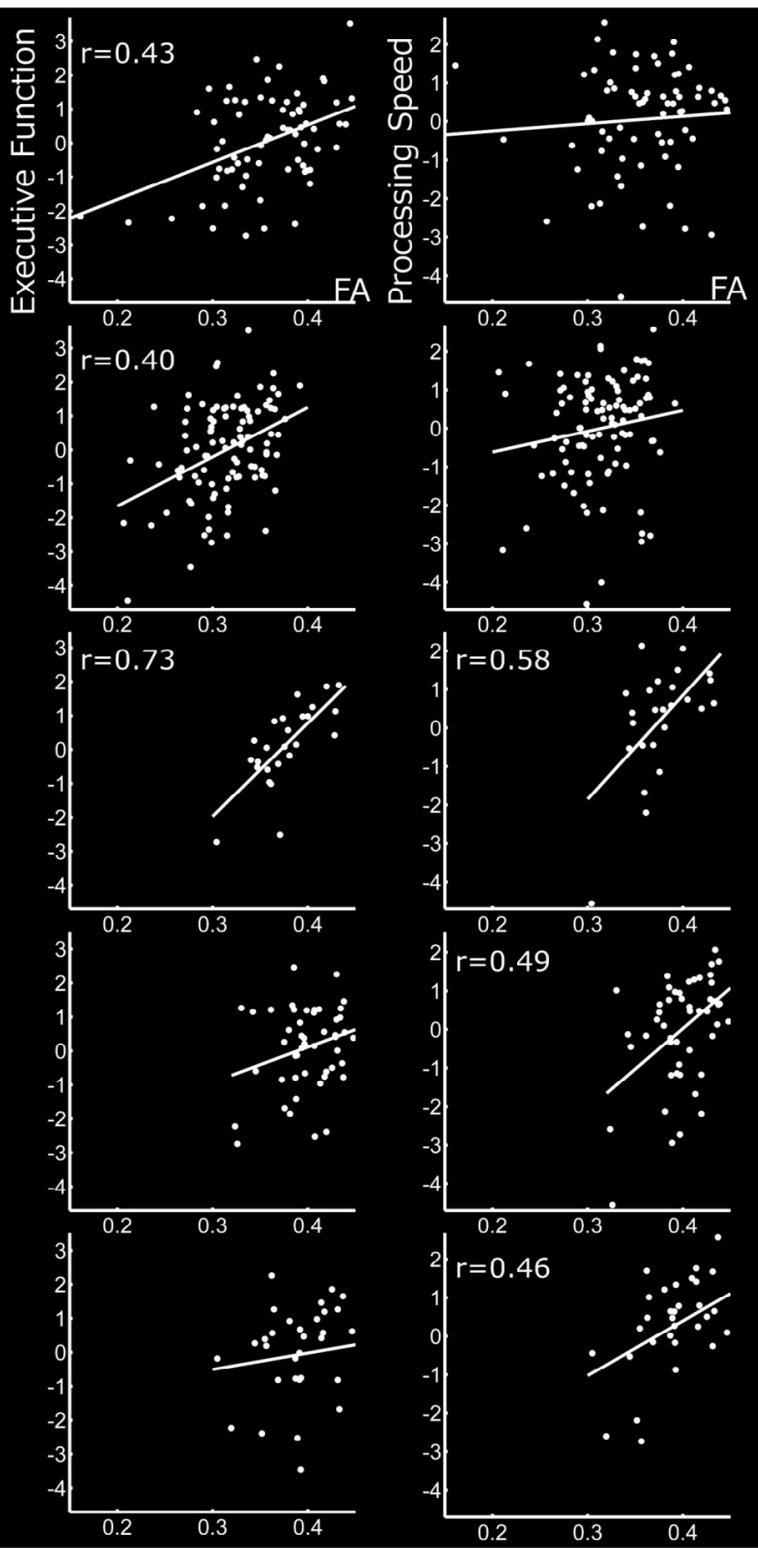

Fig. 6. Positive relationships between white matter tract integrity and cognitive performance. The left column presents $3 \mathrm{D}$ renderings of white matter tracts connecting subcortical nuclei to cortical resting state networks identified using independent components analysis (IC). Tracts include connections between thalamus (IC1) and anterior default mode network (IC6) (Row 1), inferior putamen (IC4) and anterior default mode network (Row 2), inferior putamen and dorsal attention network (IC9) (Row 3), inferior putamen and calcarine/visual/lingual cortices (IC17) (Row 4), and superior putamen (IC3) and left sensorimotor cortex (IC13) (Row 5). The number of participants displaying each connection is noted in the bottom right corner of each rendering. Scatterplots display pairwise correlations between FA from each tract and composite scores of executive function (middle column) and processing speed (right column). Least squares regression lines have been superimposed on the data, and correlation coefficients indicate significant effects at $p<0.01$. Reproduced with permission from Ystad et al. (2011).

reviews of cognitive aging revealed that, across studies, the strength of these brain-behavior relationships was weaker when analyses statistically controlled for age (Madden et al., 2009a, 2012). A handful of recent DTI studies examined relationships between white matter integrity and cognitive performance both before and after statistically controlling for age. These within-study comparisons revealed that the brain-behavior relationships were attenuated (Salami et al., 2012; Borghesani et al., 2013) or no longer significant (Metzler-Baddeley et al., 2011; Schulze et al., 2011;
Ystad et al., 2011; Lockhart et al., 2012) after accounting for age. In line with our interpretation of the between-study comparison presented in our previous review (Madden et al., 2012), this finding suggests that age, or some variable(s) associated with age, mediates relationships between white matter integrity and cognitive performance, at least in part.

Importantly, however, a majority of the recent DTI studies controlled for age, among other variables (e.g., gender, education, and white matter volume). After accounting for this age-related variance, their results still 
revealed significant relationships between white matter integrity and cognitive performance across younger and older adults (e.g., Coxon et al., 2012), lifespan samples (e.g., Bendlin et al., 2010; Salami et al., 2012; Samanez-Larkin et al., 2012; Sasson et al., 2012, 2013), and within older adults (e.g., Schiavone et al., 2009; Sexton et al., 2010; Penke et al., 2010a,b; Brickman et al., 2012; Jacobs et al., 2013). Thus, age does not fully explain relationships between white matter integrity and cognitive performance. Nonetheless, to ensure that the brain-behavior effects of interest are not compounded by age effects, future studies should examine the contribution of white matter integrity to cognitive performance after controlling for this agerelated variance. However, because chronological age often captures a large portion of age-related variance in behavioral variables, controlling for age in this way can sometimes obscure the smaller effect of white matter integrity on cognitive performance, or, more generally, decrease the sensitivity of detecting effects of brain structure (Mungas et al., 2009). An alternative is to control for the effect of chronological age on white matter, for example, by controlling for a global measure of integrity. This is especially important in light of the controversy as to whether or not age-related cognitive declines are due to localized effects of aging within specific white matter tracts or more global age-related changes shared across all white matter tracts (see the section of this article, Global versus Tract-Specific AgeRelated Effects, below).

\section{White matter integrity mediates age-behavior relationships}

Finally, our previous DTI reviews of cognitive aging revealed that the strength of relationships between age and cognitive performance were weaker when analyses statistically controlled for white matter integrity (Madden et al., 2009a, 2012). The mediating role of white matter integrity in these age-behavior relationships was further supported in a number of recent studies (Raz et al., 2005; cf., Burgmans et al., 2011; Brickman et al., 2012; Salami et al., 2012; Samanez-Larkin et al., 2012; Borghesani et al., 2013). Samanez-Larkin et al. (2012), for example, reported that, across a sample of adults 21-85 years, age was significantly associated with impaired associative learning and decreased thalamocorticostriatal tract integrity. After controlling for age, they found a positive relationship between associative learning and thalamocorticostriatal tract integrity. Importantly, the relationship between age and associative learning no longer attained significance after controlling for thalamocorticostriatal tract integrity, indicating that white matter integrity mediated the age-behavior relationship. These data are presented in Fig. 7, Panels $\mathrm{D}$ and $\mathrm{E}$. In addition to associative learning, other recent studies reported that white matter integrity mediated relationships between age and executive functions (Brickman et al., 2012; Borghesani
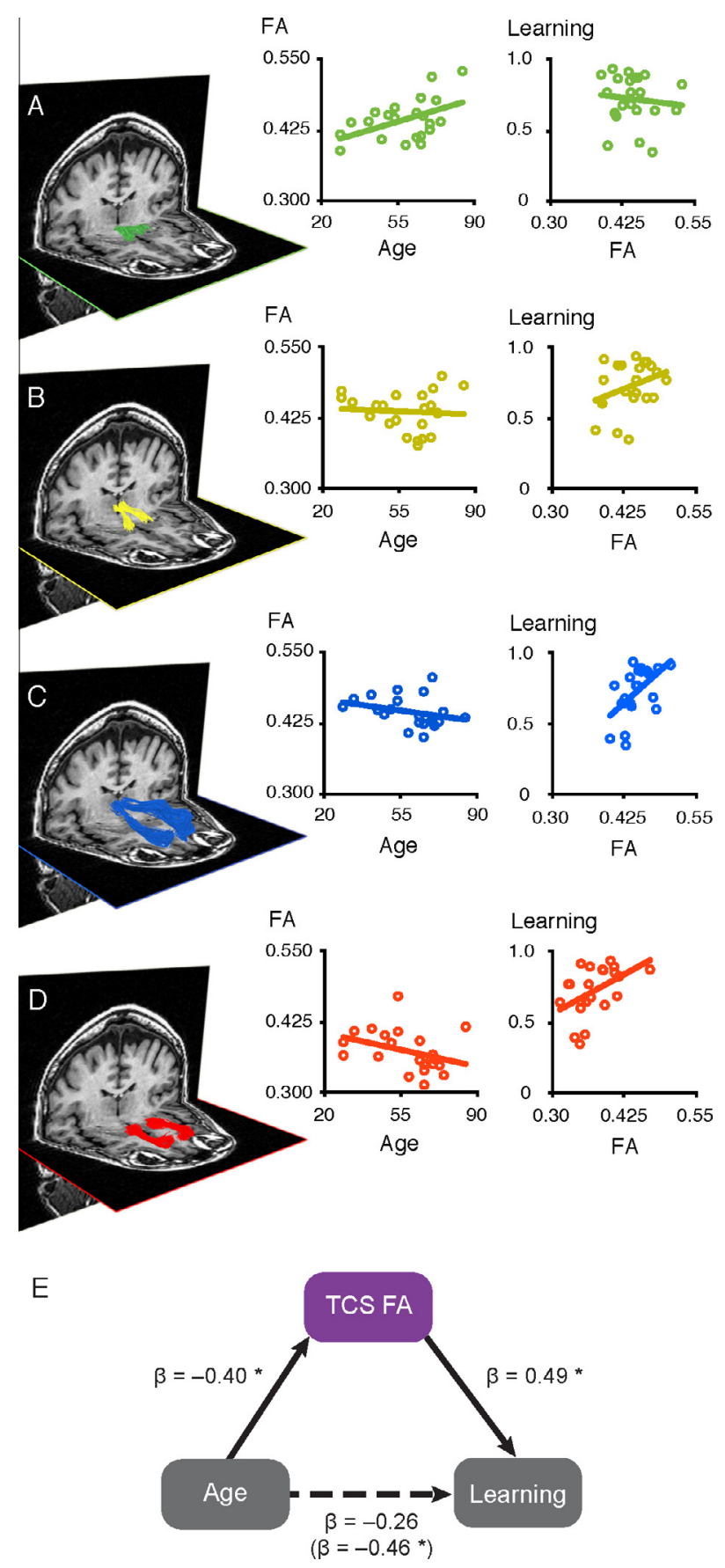

Fig. 7. Relationships among age, fronto-striatal white matter tract integrity and probabilistic reward learning. White matter tracts connecting ventral tegmental area and nucleus accumbens (Panel $A$ ), nucleus accumbens and dorsomedial thalamus (Panel B), dorsomedial thalamus and medial prefrontal cortex (Panel C), and medial prefrontal cortex and nucleus accumbens (Panel D) are presented from a representative participant. Scatter plots display pairwise correlations between FA from each tract and either age (left) or probabilistic reward learning (right). The thalamocortical (Panel C) and corticostriatal (Panel D) tracts were significantly associated with both age and learning. Furthermore, a combined measure of thalamocorticostriatal (TCS) white matter integrity significantly mediated age differences in probabilistic reward learning (Panel E). Path coefficients are standardized $\beta s$. ${ }^{*} p<0.05$, two-tailed. Reproduced with permission from Samanez-Larkin et al. (2012). 
et al., 2013) and between age and processing speed (Salami et al., 2012).

Taken together, this pattern of results is consistent with the notion that cerebral white matter integrity contributes to age-related deficits in cognitive performance. Importantly, this line of work provides the strongest evidence in support of cortical disconnection as an explanation for cognitive aging. However, these analyses do not rule out alternative relationships among these three variables, including the possibility that white matter integrity and cognitive performance are only related to each other through their relationship with age (see the section of this article, Multiple Age-BrainBehavior Models, below).

\section{CONSIDERATIONS FOR FUTURE RESEARCH}

From the DTI literature of cognitive aging reviewed above, we can conclude that (1) independently of age, increased white matter integrity is associated with better cognitive performance; (2) age mediates relationships between white matter integrity and cognitive performance, accounting for a significant portion, but not all, of the variance in these brain-behavior relationships; and (3) in some contexts, white matter integrity mediates agerelated differences in cognitive performance. However, when appropriate, we noted additional evidence indicating that our current understanding of relationships among age, white matter integrity, and cognitive performance remains limited.

These limitations highlight important directions for future research. First, it will be necessary to investigate multiple models of the age-brain-behavior relationship within a study, rather than across studies as discussed previously. Finding that white matter integrity mediates cognitive differences in aging does not rule out the possibility that white matter integrity and cognitive performance are independent and only related to each other through their relationship with age. Second, it has not yet been determined whether age-related differences in white matter integrity represent effects associated with individual tracts, or instead are global effects occurring across the whole brain. Third, the relative contribution of microstructural versus macrostructural [e.g., white matter lesions (WML)] variation in white matter integrity to age-related differences in cognition has not been resolved. Fourth, additional research using information processing tasks is needed to define the component processes of cognitive functions related to age-related differences in white matter integrity. Fifth, different imaging modalities, particularly resting-state and task-related fMRI, need to be combined with DTI and behavioral measures to clarify the role of white matter integrity in cognitive aging. In the following sections, we consider some of the implications of each of these new directions.

\section{Multiple age-brain-behavior models}

To date, DTI-based aging research has focused primarily on a particular model of the relations among aging, white matter integrity, and cognitive performance. Specifically, age-related differences in cognition are to some extent attributed to age-related declines in brain structure or function, such as white matter integrity. Salthouse (2011) proposed that our limited understanding of the neuroanatomical substrates of cognitive aging can be improved, however, by devoting greater effort to testing alternative models of relationships among age, brain, and cognitive variables. A schematic of alternative models is presented in Fig. 8. The modal model, consistent with disconnection theories of cognitive aging, is that white matter integrity mediates the relation between age and cognition (Fig. 8, model 1). As discussed in the previous section, White Matter Integrity Mediates Age-Behavior Relationships, the existing DTI literature of cognitive aging provides some support for this brain-mediating model, finding that relationships between age and cognitive performance are attenuated after controlling for white matter integrity (Perry et al., 2009; Madden et al., 2009b; Gold et al., 2010; Bennett et al., 2011; Brickman et al., 2012; Salami et al., 2012; Samanez-Larkin et al., 2012; Borghesani et al., 2013).

There are, however, alternative relationships among age, brain, and cognitive variables. For example, cognitive or behavioral variables could be mediating the relation between aging and brain measures (e.g., white matter integrity; Fig. 8, model 2). Though it may initially seem counterintuitive, this type of model underlies research on the effects of cognitive or behavioral training interventions (Salthouse, 2006; Kramer and

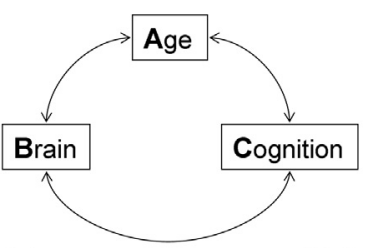

(1) $B$ as mediator

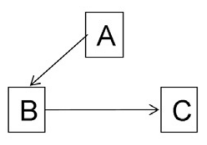

(3) Independent effects

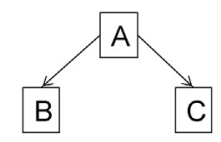

(2) $C$ as mediator

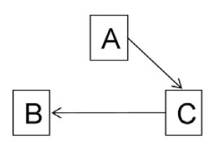

(4) $X$ as third variable

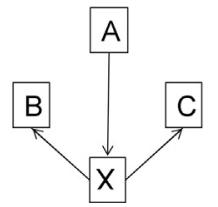

Fig. 8. Schematic illustration of relationships among age (A), brain (B), and cognitive (C) variables (top). Four alternative models of correlations among these variables are presented, including a (1) brainmediating model, in which white matter integrity mediates relationships between age and cognitive performance; (2) cognition-mediating model, in which cognitive performance mediates relationships between age and white matter integrity; (3) independent-variable model, in which white matter integrity and cognitive performance are independent and only related to each other through their relationship with age; and (4) additional-variable model, in which age, white matter integrity, and cognitive performance are related to each other through their relation to an unidentified third variable. Reproduced with permission from Salthouse (2011). 
Erickson, 2007; Lustig et al., 2009; Engvig et al., 2010; Mackey et al., 2012). According to another, independent-variable model (Fig. 8, model 3), white matter integrity and cognitive performance may instead be independent and only related to each other through their relationship with age. That is, individual differences in both white matter integrity and cognitive performance may be associated with age, without white matter integrity accounting for additional variance in performance beyond that explained by age. Some findings from previous studies are consistent with this model, specifically results indicating that relationships between white matter integrity and cognitive performance are reduced (or no longer statistically significant) after controlling for age (Metzler-Baddeley et al., 2011; Schulze et al., 2011; Ystad et al., 2011; Lockhart et al., 2012; Salami et al., 2012; Borghesani et al., 2013; Johnson et al., 2013). Finally, it is possible that age, white matter integrity, and cognitive performance are only related to each other as a result of their relation to some additional, unidentified variable (Fig. 8, model 4). The relevant variable may be an index of health status, lifestyle variable, or genetic risk factors, which often remains unmeasured or uncontrolled. This type of model has received less attention in the field, but is potentially important.

Thus, Salthouse (2011) proposed that advances in understanding the neuroanatomical substrates of cognitive aging depend on the comparison of alternative models rather than just testing the success of individual models. Whereas the brain-mediating model traditionally guides studies that aim to identify white matter integrity correlates of cognitive aging, the independent-variable model is probed by studies interested in (age-invariant) white matter integrity correlates of cognition. In order to strengthen their conclusion, however, future studies would need to contrast these models by assessing both age-behavior relationships with and without controlling for white matter integrity (test for the brain-mediating model) and brain-behavior relationships after control for age-related variance (test for the independent-variable model). But in this latter case, if the goal is to detect specific relations between brain-related variables and cognitive performance, any adjustments in the outcome variable(s) as a function of the age variable may decrease the sensitivity of the detection of brain-related effects (Mungas et al., 2009). At a theoretical level, comparison of the alternative models provides more compelling evidence regarding the nature of relationships among the age, brain, and cognitive variables, but this benefit must be evaluated within the larger context of the research goals.

Taken together, emerging data appear more consistent with the independent-variable model relative to the brain-mediation model. Three recent studies, for example, directly contrasted the brain-mediating and independent-variable models within the same dataset (Zahr et al., 2009; Salami et al., 2012; Borghesani et al., 2013), and all three studies found that relationships between white matter integrity and cognitive performance were reduced or no longer significant after controlling for age, consistent with the independentvariable model. The three studies differed, however, in their support for the brain-mediation model. Whereas Salami et al. (2012) provided unequivocal support for the independent-variable model, Zahr et al. (2009) and Borghesani et al. (2013) also found support for the brain-mediating model, in that relationships between age and cognitive performance were reduced or no longer significant after controlling for white matter integrity. Interestingly, however, Zahr et al. reported that age attenuated brain-behavior relationships to a similar degree as white matter integrity attenuated agebehavior relationships ( $>82 \%$ ), suggesting that only a small proportion of the variance in performance could be uniquely attributed to either age or white matter integrity. Thus, although a brain-mediation model may provide the best theoretical framework for cortical disconnection, and an appropriate starting point for theory development, alternative models should always be considered and explored, within the same data set when possible.

\section{Global versus tract-specific age-related effects}

Neuroimaging studies of cerebral white matter integrity frequently compare individual tracts or regions of interest, under the assumption that different anatomical regions may contribute differentially to age-related differences in cognitive function. Although this assumption may often be justified, it is an empirical question whether, within a particular data set, the uniquely age-related effects associated with individual regions can be reliably distinguished from their shared effects. A similar issue exists in behavioral investigations of cognitive aging, particularly those measuring the speed of response. For example, Salthouse (1985) pointed out that reaction time will typically be higher overall for older adults than for younger adults, for a variety of reasons including declines in sensorimotor functioning (Birren, 1965; Cerella, 1985; Schneider and Pichora-Fuller, 2000). Thus, when statistical analyses reveal an interaction with age group, it is important to distinguish between age-related differences that are associated with the cognitive demands of specific task conditions and those that are associated with more general, shared effects (i.e., age-related slowing). Behavioral investigations of cognitive aging have addressed this issue by using various data analytic approaches, including transformations of reaction time, so that the influence of general age-related slowing may be taken into account (Salthouse, 1996; Faust et al., 1999; Madden, 2001; Salthouse and Madden, 2007).

In the case of DTI measures, the question is whether age-related differences for individual tracts or regions of interest can be interpreted independently of more global, whole-brain age effects. Increasing adult age may be associated with some change in white matter integrity throughout the brain, and thus selecting individual tracts for analysis may be essentially sampling from this general effect. Even in the absence of a truly general 
effect, the data for individual tracts will be correlated to some extent, particularly tracts that are spatially contiguous. Therefore, by analyzing each tract individually, the contribution from the shared variance across tracts is not taken into account. To date, research on this issue has been limited. Thus, in characterizing the relation between white matter integrity and age-related differences in cognitive performance, methods for distinguishing between whole-brain (global) and tract-specific effects will be valuable.

Penke et al. (2010b) have demonstrated a prominent influence of a global effect of white matter integrity in older adults. These authors used principal component analysis (PCA), which identifies latent (component) variables that are responsible for the shared variance among manifest (measured) variables. The PCA was applied to each of several integrity measures (FA, AD, $\mathrm{RD}$, and $\mathrm{MD}$ ), from eight white matter tracts in a sample of 132 older adults. For each integrity measure, all of the individual tracts (except the splenium) loaded highly on the first component, which explained approximately $45 \%$ of the variance in each measure. Two of these global components (those for FA and RD) were correlated with information processing speed, but not with general intelligence or memory. The Penke et al. results suggest that, among older adults, individual differences in white matter integrity are a relatively global effect across nearly all of the selected white matter tracts. This global effect, however, has a specific relation to one form of fluid cognition (informationprocessing speed).

Lovden et al. (2013) also obtained some evidence for a global effect of white matter integrity in a sample of 260 older adults. These authors examined integrity (FA, MD) from seven white matter tracts in each hemisphere using structural equation modeling to compare two models: one positing a general factor (i.e., individual differences shared across all tracts) and one modeling tract-specific effects (i.e., individual differences shared between homologous pairs of tracts). Consistent with the Penke et al. (2010b) findings, a global factor was evident in the data. However, the model that explained the most variance contained specific factors for homologous tract pairs, thus supporting a tract-specific view. Also supportive of a tract-specific account, Li et al. (2012) conducted an independent component analysis of whole-brain FA maps and found that multiple independent components emerged, which corresponded to groups of white matter tracts (e.g., supratentorial commissural, association, or projection pathways).

Thus, some evidence exists for both global and tractspecific individual differences in cerebral white matter integrity, although how these patterns of individual differences are related to age-related variance in cognitive performance is not yet entirely clear. Whereas Penke et al. (2010b) and Lovden et al. (2013) focused on samples of older adults (71-73 and 60-87, respectively), Li et al.(2012) used a wider range of middle-aged adults (20-50 years). Westlye et al. (2010) examined several DTI measures in 430 individuals across a wide range of $8-85$ years of age, but the goal was to characterize developmental trajectories rather than the differentiation of global and tract-specific effects. Tamnes et al. (2011) found that PCA yielded evidence for a global factor, across 64 brain regions, within each of several structural brain measures (cortical thickness, white matter volume, FA, and MD) for 168 participants. Further, these global components correlated significantly with a measure of general intellectual ability. However, Tamnes et al. were concerned with cognitive development in younger individuals 8-30 years of age.

Johnson et al. (2013) addressed the age-related differences in both DTI measures of white matter integrity and behavioral measures of cognition, in 52 younger adults (18-28 years) and 64 older adults (6085 years). These authors, using a PCA methodology similar to that of Penke et al. (2010b), identified latent variables from white matter integrity across eight regions corresponding to anatomically defined white matter tracts. The data yielded some evidence for a global effect beyond the level of individual tracts, in that analyses that combined both age groups identified latent components representing groups of tracts. However, individual tracts varied in their contribution to this global component. Thus, the results represented a mixture of global and tract-specific effects, similar to Li et al.(2012), rather than the entirely global effects as proposed by Penke et al. Further, Johnson et al. conducted mediation analyses that demonstrated, in this data set, that age mediated the relation between one of the FA components and sensorimotor speed (digit symbol RT), but the FA component was not a mediator of the agespeed relation. Thus, some variable associated with age but not measured, such as cardiovascular risk factors, led to an association between decreasing FA and decreasing sensorimotor speed. More generally, the Johnson et al. findings reinforce the Salthouse (2011) recommendation that comparing different models of the relations among age, cognition, and neuroimaging variables is needed to define which variable (if any) is a mediator within a particular data set.

\section{WML}

In this review, we have focused on DTI measures that represent the microstructural organization of white matter, as inferred from the rate and directionality of diffusion of molecular water. Further, in the majority of the relevant studies, the DTI measures are limited to normal-appearing white matter, that is, undamaged tissue. With increasing adult age, however, macrostructural changes in white matter, in the form of decreasing volume (atrophy) and degeneration of white matter (lesions), also increase (de Leeuw et al., 2001). WML are evident as hyperintense areas in T2-weighted or proton density imaging, particularly fluid-attenuated inversion recovery (FLAIR) sequences, which can suppress cerebrospinal fluid effects on the image (Gawne-Cain et al., 1997). As shown in Fig. 9, WML are prominent in periventricular regions. Various forms of pathophysiology lead to WML but a primary factor 


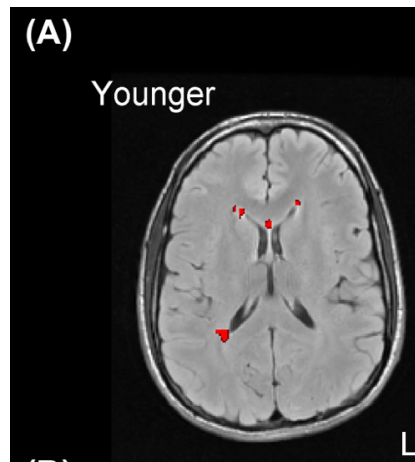

\section{(B)}
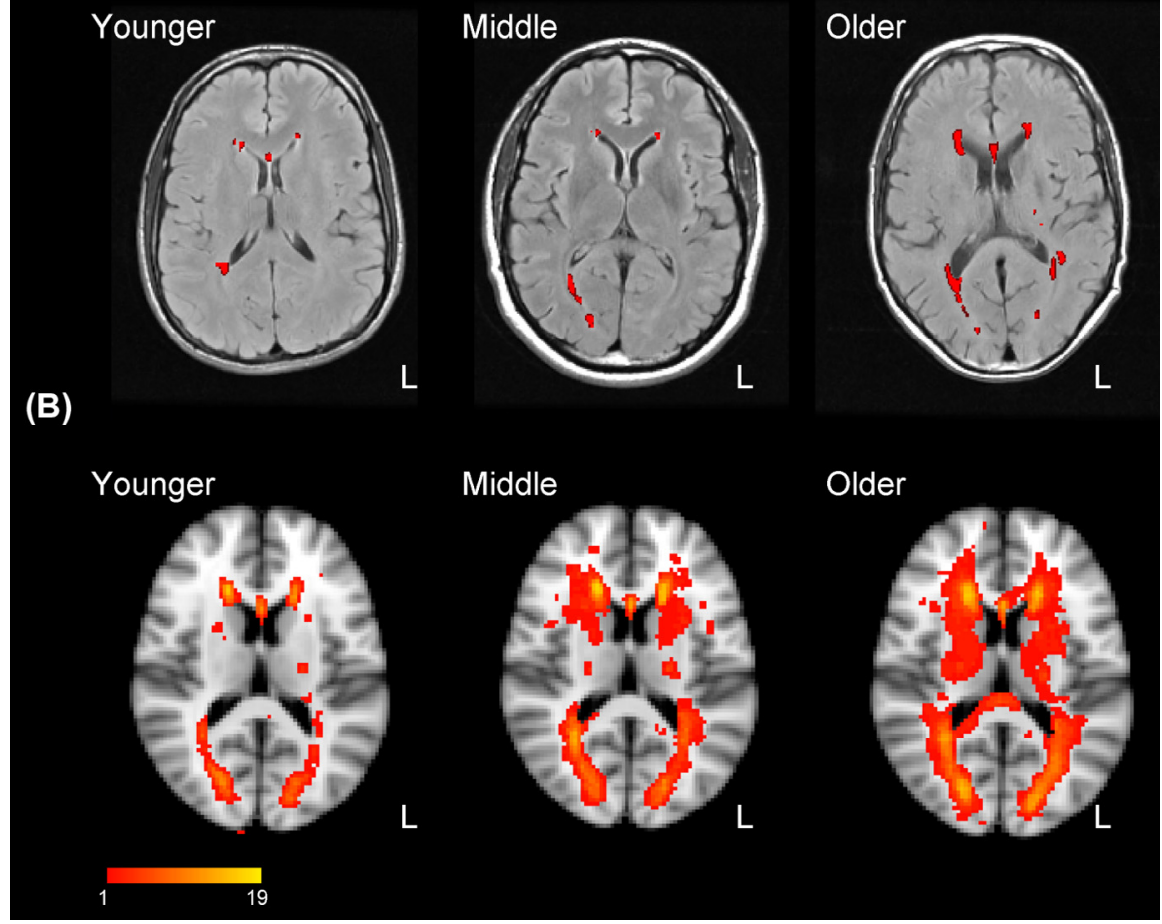

Fig. 9. White matter lesion volume. Panel A: White matter lesions for individual adults 20,48 , and 65 years of age, in T2-weighted FLAIR images. Participants were healthy, community-dwelling individuals without any sign of cognitive impairment on neuropsychological testing or history of cardiovascular disease (other than hypertension). Lesions, as identified from a semi-automated program separating lesions from normal white matter appear in red. Panel B: Composite lesion maps for 23 younger adults (19-39 years of age), 19 middle-aged adults (40-59 years of age), and 16 older adults (60-79 years of age), overlaid on a T1-weighted template. Images are in radiological orientation (left $=$ right). Color scale represents the number of individuals within each group exhibiting a lesion, per voxel. Authors' unpublished data.

appears to be cerebrovascular changes leading to vessel dysfunction and consequently an ischemic environment (Young et al., 2008; Salat, 2011). Typically, in DTI studies, image thresholding techniques are used to separate normal-appearing and lesioned white matter, and data analyses are limited to one tissue class or the other. However, the distinction between normalappearing but low FA tissue and a frank lesion is not entirely clear-cut. For a particular region, FA decreases as a function of spatially proximate WML, and lesions may represent the foci or penumbra of more subtle and widespread white matter changes, rather than distinct anatomic abnormalities (Maillard et al., 2011).

Age-related increases in WML are to some extent a phenomenon of normal aging, as is some degree of white matter and gray matter atrophy, in that these macrostructural changes can occur in the context of otherwise good health and preserved cognitive function. But increasing WML volume is also correlated with impairments in aspects of cognition (Vernooij et al., 2009; Smith et al., 2011; Maillard et al., 2012; Valdes Hernandez et al., 2013). At a systems level, the effects of macrostructural damage from WML are similar to decreases in the microstructural integrity of normalappearing white matter: a disconnection of the networks of cortical regions necessary for cognition. Thus, although our focus in this review is on DTI analyses of normal-appearing white matter, a complete account of cerebral white matter integrity and cognitive aging must also consider the potential role of WML.

For example, it will be important for future research to clarify relationships between white matter integrity and WML. In a population-based study with 832 healthy older adults, Vernooij et al. (2008) compared the agerelated differences in white matter integrity, atrophy, and WML. Age-related differences associated with white matter integrity were minimal when atrophy and WML were taken into account. Further, atrophy and WML exhibited correlations with FA in different regions, suggesting that these two variables are distinct in pathophysiology. The DTI analysis, however, used a normalized approach (tract-based spatial statistics; TBSS; Smith et al., 2006), in which analyses were conducted using data from the center of tracts common to all participants (i.e., a skeletonized FA map). While this approach provides superior between-subject registration and is less subject to partial volume effects, focusing on the center of white matter tracts may not fully capture inter-subject variability. Thus, while the Vernooij et al. analyses suggest that age-related decline in the microstructural integrity of white matter may be explained primarily by atrophy and lesion formation, this hypothesis should be explored further with other analytic techniques.

The contribution of these microstructural (white matter integrity) and macrostructural (WML, white matter 
volume) variables to cognitive aging also requires further scrutiny as some evidence suggests that WML may have a more important role in cognitive function than microstructural integrity, but the contribution of underlying pathology is unclear. Meier et al. (2012), for example, compared WML and a summary FA measure as predictors of several tests of cognitive function, in a sample comprising both cognitively impaired older adults and healthy controls. Increased WML volume in the frontal lobe was associated with poorer delayed memory performance, whereas FA was not a predictor of cognitive test performance. Meier et al. noted that in this sample, with a prominent representation of the history of vascular disease, WML may be more relevant to cognition than the microstructural integrity of healthy white matter. Parks et al. (2011) also found that WML have a mediating (causal) role in cognitive function (episodic memory and executive function) in their sample of 422 healthy older adults and individuals diagnosed with mild cognitive impairment and dementia. Further analyses revealed that the effect of WML on memory in this older and clinically diverse sample was indirect and dependent on the relation between executive function and hippocampal volume. Complementary longitudinal data suggest that the influence of WML on executive function and episodic memory, in older adults, operates through the extension of pre-existing lesions rather the emergence of new lesions (Maillard et al., 2012).

In contrast, Burgmans et al. (2011) compared microstructural and macrostructural variables as potential mediators of age-sensitive cognitive functions (executive function and perceptual-motor speed) in 219 healthy older adults. Their most critical result was that, whereas an age-related increase in WML volume was significant, as was age-related decline in all of the anisotropy measures, only linear anisotropy (related more closely to axonal integrity) was a mediator of the relation between age and the cognitive measures (processing speed). The presence of hypertensive participants did not affect the results. Thus, in contrast to the Vernooij et al. (2008) findings, the relation between age-related differences in cognition and white matter were more clearly evident in the microstructural indices of healthy white matter than in the WML volume. Burgmans et al. concluded that in healthy adults, deterioration of axonal integrity (through axonal damage and demyelination of white matter) and the ensuing breech of connectivity may contribute to age-related slowing of information processing.

\section{General versus task-specific components of performance}

We have noted previously that age-related differences in behavioral measures of cognitive performance have general (i.e., age-related slowing) as well as taskspecific components. In developing models of the relations among aging, cognition, and white matter integrity, it will be important to recognize that the models may differ as a function of the way in which cognition is measured. General measures, for example, are typically obtained by combining data from psychometric tests that are different instances of a common cognitive domain (e.g., perceptual-motor speed, executive function, and memory), which can be done either on the basis of a pre-existing theoretical framework (Andrews-Hanna et al., 2007) or on a more data-driven basis, from factor analytic techniques that identify latent variables from manifest measures (Burgmans et al., 2011; Haasz et al., 2013; Sasson et al., 2013). Analyses relying on one or more general measures of cognition have several positive features, including standardization of procedures across testing environments and the removal of measurement error and task-specific variance.

Alternatively, task-specific variance may be the aspect of the data that yields the most theoretically informative pattern of age-related effects. Several DTI aging studies have used information-processing tasks that measure the time required for specific cognitive operations such as semantic memory retrieval (Madden et al., 2009b) and switching between task sets (Perry et al., 2009; Gold et al., 2010). Bennett et al. (2012), for example, used a visual search task that varied the attentional demands between a relatively simple feature discrimination (detection of a green, upright $\mathrm{T}$ target among red distractor Ts) or more complex discrimination of conjunctions of features (detection of a green, upright $\mathrm{T}$ target among red, upright $\mathrm{Ts}$ and green, inverted Ts). For both younger and older adults, higher integrity of frontoparietal white matter tracts (i.e., genu and splenium of corpus callosum, superior and inferior longitudinal fasciculi) was correlated with lower visual search reaction time (i.e., faster responding). When overall motor speed was controlled statistically, however, only FA from the genu and left superior longitudinal fasciculus were significant predictors of search reaction time. Thus, by isolating the components of performance specific to visual search, Bennett et al. were able to identify the most relevant tracts within the frontoparietal network.

This task-specific approach has the advantage of increasing the specificity of the cognitive outcome variable. It is often useful to retain the metric of real time as a dependent measure (Pachella, 1974). In sum, task-specific analyses will typically be more valuable in smaller sample studies that have specific hypotheses regarding the cognitive processes most relevant to white matter integrity, whereas more general measures of cognitive domains will be valuable in larger sample studies that do not require defining the individual stages of information processing within a task.

\section{Multimodal imaging}

Beyond the microstructural and macrostructural effects in cerebral white matter integrity, aging is associated with other structural changes such as gray matter atrophy and ventricular enlargement (Anderton, 2002; Raz et al., 2005), as well as functional changes in the form of increased and decreased regional cortical activation and functional connectivity (Dennis and Cabeza, 2008; Grady, 2008; Park and Reuter-Lorenz, 2009; Parks and 
Madden, 2013). Thus, disconnected aging, as discussed here, likely results from a combination of structural and functional aspects of brain aging. Future research combining DTI with additional neuroimaging techniques, especially functional MRI (fMRI), will be necessary to provide a more complete understanding of how brain structure and brain function contribute to cognition in aging. Multimodal imaging will also have the benefit of moving interpretations of neural data from functional segregation (i.e., regional localization) to functional integration (i.e., information flow between networks; Ramnani et al., 2004).

Initial multimodal imaging studies in aging combined DTI measures of structural integrity with $\mathrm{FMRI}$ measures of intrinsic functional connectivity. Intrinsic functional connectivity refers to covariation in the low-frequency $(<0.10 \mathrm{~Hz})$ component of the fMRI signal time course that is evident either in the absence of a task (i.e., resting state) or in analyses that attenuate taskdependent components of the fMRI signal (Fox and Raichle, 2007; Biswal et al., 2010; Yeo et al., 2011). For example, Andrews-Hanna et al. (2007) reported that healthy older adults exhibited a decrease in intrinsic functional connectivity relative to younger adults, and that, within the older group, reduced connectivity was associated with both reduced white matter integrity and behavioral measures of cognitive performance. Chen et al. (2009) similarly found that increasing intrinsic functional connectivity within primarily prefrontal regions was associated with lower choice reaction time (i.e., faster responding) in a group of healthy older adults. Further, $87 \%$ of the age-related variance in choice reaction time was shared with individual differences in functional connectivity. The older adults' intrinsic functional connectivity, in turn, was correlated positively with FA in the genu of the corpus callosum, consistent with a disconnection model.

Also consistent with the notion of cortical disconnection, Davis et al. (2012) compared unilateral and bilateral presentation in a word-matching task and found a performance advantage to bilateral presentation that was greater in magnitude for older adults than for younger adults. Importantly, older adults also exhibited greater functional connectivity between left and right prefrontal regions, during the bilateral condition, than younger adults, and older adults' task-related connectivity was correlated positively with both FA and $\mathrm{RD}$ in the genu of the corpus callosum. However, in a study of task-related functional connectivity during an executive control task (task-switching), Madden et al. (2010) found that although functional connectivity significantly accounted for a portion of the age-related variance in semantic categorization performance, the integrity of frontal and posterior white matter (genu and splenium of the corpus callosum, respectively) did not make age-independent contributions to functional connectivity. The potential influence of several variables, such as the difference between intrinsic and task-related functional connectivity, and the magnitude and direction of age-related differences in the imaging and behavioral variables, remain to be explored in further investigations of age-related differences in cerebral white matter integrity.

A growing body of literature has also combined DTI and fMRI research to study the functional neuroanatomy of cognitive aging (Bennett and Rypma, 2013). In support of cortical disconnection, these studies reveal that healthy aging is accompanied by declines in the integrity of white matter connections between brain regions and disrupted processing within those regions (i.e., age-related increases and decreases neural activity), both of which contribute to age-related cognitive declines. Schulze et al. (2011), for example, acquired DTI and fMRI during performance of a working memory (n-back) task in healthy younger and older adults. These authors found that, relative to younger adults, older adults showed declines in working memory performance, decreased white matter integrity (wholebrain and prefrontal), and both increased (especially in frontal regions) and decreased (especially in visual regions) task-dependent activation. A series of correlations further supported these relationships, revealing that decreased frontal white matter integrity was significantly related to increased age, decreased working memory performance, and decreased frontal activity. The positive relationship between frontal white matter integrity and frontal activity remained significant even after controlling for age. However, others have observed negative relationships between frontal white matter integrity and frontal activity during different task conditions (e.g., Zhu et al., 2013). These data are consistent with a disconnection model, but as with the Andrews-Hanna et al. (2007) and Chen et al. (2009) results, the evidence is indirect because alternative models were not compared directly.

More recently, a number of multimodal imaging studies of cognitive aging have employed three or more neuroimaging approaches (Burgmans et al., 2011; Schulze et al., 2011; Ystad et al., 2011; He et al., 2012). In addition to collecting DTI and fMRI data, for example, Schulze et al. (2011) acquired volumetric data using structural MRI. Their results revealed that white matter volume was not significantly related to their measures of white matter integrity or neural activity. Such data sets are complex, and the detection of subtle interactions among the variables of interest requires large sample sizes and often advanced statistical approaches. Nonetheless, future multimodal imaging research that combines two or more neuroimaging techniques will be an integral part to revealing how brain structure (white matter integrity, volume) and brain function (neural activity, functional connectivity) contribute to cognition, and whether these brainbehavior relationships contribute to cognitive aging as proposed by cortical disconnection theories.

\section{CONCLUSIONS}

Findings from recent DTI studies of cognitive aging lead to several conclusions: (1) White matter integrity typically declines in healthy aging, as reflected in agerelated decreases in FA and increases in MD. (2) These 
age effects are likely driven by changes in underlying myelin, as suggested by preferential age-related increases in RD relative to AD. (3) Increased white matter integrity relates to better cognitive performance, that is, the age-independent relationships between white matter integrity and cognitive performance are predominantly positive. (4) Statistically controlling for chronological age typically weakens brain-behavior relationships, suggesting that age mediates the effect of white matter integrity on cognitive performance. (5) In some contexts, white matter integrity mediates agerelated declines in cognitive performance, as seen by weaker age-behavior relationships when analyses statistically control for white matter integrity. Taken together, these findings are generally consistent with the notion that healthy aging is accompanied by cortical disconnection, and that, in turn, these age-related differences in white matter integrity contribute to the different forms of cognitive declines observed in healthy older adults.

As discussed throughout the current review, however, a number of questions remain unanswered regarding the nature of relationships among white matter integrity, cognitive performance, and aging. Specifically, additional support for cortical disconnection interpretations will require that future studies pursue several new avenues: (1) exploring multiple models of age-brain-behavior relationships; (2) differentiating global and tract-specific effects; (3) assessing the potential contribution of WML; (4) isolating component processes of cognition using information processing tasks; and (5) combining information from structural and functional imaging modalities.

Acknowledgements-Preparation of this manuscript was supported by NIH research grant R01 AG039684. We are grateful to Sally Cocjin, David Hoagey, Ying-hui Chou, and Emily Parks for their assistance.

\section{REFERENCES}

Aboitiz F, Rodriguez E, Olivares R, Zaidel E (1996) Age-related changes in fibre composition of the human corpus callosum: sex differences. Neuroreport 7:1761-1764.

Anderton BH (2002) Ageing of the brain. Mech Ageing Dev 123:811-817.

Andrews-Hanna JR, Snyder AZ, Vincent JL, Lustig C, Head D, Raichle ME, Buckner RL (2007) Disruption of large-scale brain systems in advanced aging. Neuron 56:924-935.

Antonenko D, Meinzer M, Lindenberg R, Witte AV, Floel A (2012) Grammar learning in older adults is linked to white matter microstructure and functional connectivity. Neuroimage 62:1667-1674.

Baron RM, Kenny DA (1986) The moderator-mediator variable distinction in social psychological research: conceptual, strategic, and statistical considerations. J Pers Soc Psychol 51:1173-1182.

Barrick TR, Charlton RA, Clark CA, Markus HS (2010) White matter structural decline in normal ageing: a prospective longitudinal study using tract-based spatial statistics. Neuroimage 51:565-577.

Bartzokis G (2004) Age-related myelin breakdown: a developmental model of cognitive decline and Alzheimer's disease. Neurobiol Aging 25:5-18.
Basser PJ, Mattiello J, LeBihan D (1994) MR diffusion tensor spectroscopy and imaging. Biophys J 66:259-267.

Beaulieu C (2002) The basis of anisotropic water diffusion in the nervous system - a technical review. NMR Biomed 15:435-455.

Bendlin BB, Fitzgerald ME, Ries ML, Xu G, Kastman EK, Thiel BW, Rowley HA, Lazar M, Alexander AL, Johnson SC (2010) White matter in aging and cognition: a cross-sectional study of microstructure in adults aged eighteen to eighty-three. Dev Neuropsychol 35:257-277.

Bennett IJ, Rypma B (2013) Advances in functional neuroanatomy: a review of combined DTI and fMRI studies in healthy younger and older adults. Neurosci Biobehav Rev 37:1201-1210.

Bennett IJ, Motes MA, Rao NK, Rypma B (2012) White matter tract integrity predicts visual search performance in young and older adults. Neurobiol Aging 33:433.e421-433.e431.

Bennett IJ, Madden DJ, Vaidya CJ, Howard DV, Howard Jr JH (2010) Age-related differences in multiple measures of white matter integrity: a diffusion tensor imaging study of healthy aging. Hum Brain Mapp 31:378-390.

Bennett IJ, Madden DJ, Vaidya CJ, Howard Jr JH, Howard DV (2011) White matter integrity correlates of implicit sequence learning in healthy aging. Neurobiol Aging 32:2317.e2311-2317.e2312.

Bhagat YA, Beaulieu C (2004) Diffusion anisotropy in subcortical white matter and cortical gray matter: changes with aging and the role of CSF-suppression. J Magn Reson Imaging 20:216-227.

Birren JE (1965) Age changes in speed of behavior: its central nature and physiological correlates. In: Welford AT, Birren JE, editors. Behavior, aging, and the nervous system. Springfield, IL: Thomas.

Biswal BB et al (2010) Toward discovery science of human brain function. Proc Natl Acad Sci USA 107:4734-4739.

Borghesani PR, Madhyastha TM, Aylward EH, Reiter MA, Swarny BR, Warner Schaie K, Willis SL (2013) The association between higher order abilities, processing speed, and age are variably mediated by white matter integrity during typical aging. Neuropsychologia 51:1435-1444.

Bosch B, Arenaza-Urquijo EM, Rami L, Sala-Llonch R, Junque C, Sole-Padulles C, Pena-Gomez C, Bargallo N, Molinuevo JL, Bartres-Faz D (2012) Multiple DTI index analysis in normal aging, amnestic $\mathrm{MCl}$ and $\mathrm{AD}$. Relationship with neuropsychological performance. Neurobiol Aging 33:61-74.

Brickman AM, Meier IB, Korgaonkar MS, Provenzano FA, Grieve SM, Siedlecki KL, Wasserman BT, Williams LM, Zimmerman ME (2012) Testing the white matter retrogenesis hypothesis of cognitive aging. Neurobiol Aging 33:1699-1715.

Bucur B, Madden DJ, Spaniol J, Provenzale JM, Cabeza R, White LE, Huettel SA (2008) Age-related slowing of memory retrieval: contributions of perceptual speed and cerebral white matter integrity. Neurobiol Aging 29:1070-1079.

Burgmans S, Gronenschild EH, Fandakova Y, Shing YL, van Boxtel MP, Vuurman EF, Uylings HB, Jolles J, Raz N (2011) Age differences in speed of processing are partially mediated by differences in axonal integrity. Neuroimage 55:1287-1297.

Burzynska AZ, Preuschhof C, Backman L, Nyberg L, Li SC, Lindenberger U, Heekeren HR (2010) Age-related differences in white matter microstructure: region-specific patterns of diffusivity. Neuroimage 49:2104-2112.

Carmichael O, Lockhart S (2012) The role of diffusion tensor imaging in the study of cognitive aging. Curr Top Behav Neurosci 11:289-320.

Catani M (2006) Diffusion tensor magnetic resonance imaging tractography in cognitive disorders. Curr Opin Neurol 19:599-606.

Catani M, Ffytche DH (2005) The rises and falls of disconnection syndromes. Brain 128:2224-2239.

Cerella J (1985) Information processing rates in the elderly. Psychol Bull 98:67-83.

Charlton RA, Barrick TR, Markus HS, Morris RG (2009) Theory of mind associations with other cognitive functions and brain imaging in normal aging. Psychol Aging 24:338-348.

Charlton RA, Landau S, Schiavone F, Barrick TR, Clark CA, Markus HS, Morris RG (2010) Up the Garden Path: a critique of Penke 
and Deary (in press) and further exploration concerning the Charlton e al. (2008) path analysis relating loss of white matter integrity to cognition in normal aging. Neurobiol Aging 31:1661-1666.

Charlton RA, Barrick TR, McIntyre DJ, Shen Y, O'Sullivan M, Howe FA, Clark CA, Morris RG, Markus HS (2006) White matter damage on diffusion tensor imaging correlates with age-related cognitive decline. Neurology 66:217-222.

Chen NK, Chou YH, Song AW, Madden DJ (2009) Measurement of spontaneous signal fluctuations in fMRI: adult age differences in intrinsic functional connectivity. Brain Struct Funct 213:571-585.

Coxon JP, Van Impe A, Wenderoth N, Swinnen SP (2012) Aging and inhibitory control of action: cortico-subthalamic connection strength predicts stopping performance. J Neurosci 32:8401-8412.

Craik FIM, Salthouse TA, editors. The handbook of aging and cognition. New York: Psychology Press.

Davis SW, Kragel JE, Madden DJ, Cabeza R (2012) The architecture of cross-hemispheric communication in the aging brain: linking behavior to functional and structural connectivity. Cereb Cortex 22:232-242.

Davis SW, Dennis NA, Buchler NG, White LE, Madden DJ, Cabeza R (2009) Assessing the effects of age on long white matter tracts using diffusion tensor tractography. Neuroimage 46:530-541.

de Leeuw FE, de Groot JC, Achten E, Oudkerk M, Ramos LM, Heijboer R, Hofman A, Jolles J, van Gijn J, Breteler MM (2001) Prevalence of cerebral white matter lesions in elderly people: a population based magnetic resonance imaging study. The Rotterdam Scan Study. J Neurol Neurosurg Psychiatry 70: 9-14.

Dennis NA, Cabeza R (2008) Neuroimaging of healthy cognitive aging. In: Craik FIM, Salthouse TA, editors. The handbook of aging and cognition. New York: Psychology Press. p. 1-54.

Douaud G, Jbabdi S, Behrens TE, Menke RA, Gass A, Monsch AU, Rao A, Whitcher B, Kindlmann G, Matthews PM, Smith S (2011) DTI measures in crossing-fibre areas: increased diffusion anisotropy reveals early white matter alteration in $\mathrm{MCl}$ and mild Alzheimer's disease. Neuroimage 55:880-890.

Engvig A, Fjell AM, Westlye LT, Moberget T, Sundseth O, Larsen VA, Walhovd KB (2010) Effects of memory training on cortical thickness in the elderly. Neuroimage 52:1667-1676.

Ennis DB, Kindlmann G (2006) Orthogonal tensor invariants and the analysis of diffusion tensor magnetic resonance images. Magn Reson Med 55:136-146.

Faust ME, Balota DA, Spieler DH, Ferraro FR (1999) Individual differences in information-processing rate and amount: implications for group differences in response latency. Psychol Bull 125:777-799.

Filley CM (2005) White matter and behavioral neurology. Ann NY Acad Sci 1064:162-183.

Fjell AM, Walhovd KB (2010) Structural brain changes in aging: courses, causes and cognitive consequences. Rev Neurosci 21:187-221.

Forstmann BU, Tittgemeyer M, Wagenmakers EJ, Derrfuss J, Imperati D, Brown S (2011) The speed-accuracy tradeoff in the elderly brain: a structural model-based approach. J Neurosci 31:17242-17249.

Fox MD, Raichle ME (2007) Spontaneous fluctuations in brain activity observed with functional magnetic resonance imaging. Nat Rev Neurosci 8:700-711.

Gawne-Cain ML, Silver NC, Moseley IF, Miller DH (1997) Fast FLAIR of the brain: the range of appearances in normal subjects and its application to quantification of white-matter disease. Neuroradiology 39:243-249.

Geschwind N (1965a) Disconnexion syndromes in animals and man. I. Brain 88:237-294.

Geschwind N (1965b) Disconnexion syndromes in animals and man. II. Brain 88:585-644.

Gold BT, Powell DK, Xuan L, Jicha GA, Smith CD (2010) Age-related slowing of task switching is associated with decreased integrity of frontoparietal white matter. Neurobiol Aging 31:512-522.
Gouttard S, Prastawa M, Bullitt E, Lin W, Goodlett C, Gerig G (2009) Constrained data decomposition and regression for analyzing healthy aging from fiber tract diffusion properties. Med Image Comput Comput Assist Interv 12:321-328.

Grady CL (2008) Cognitive neuroscience of aging. Ann NY Acad Sci 1124:127-144.

Haasz J, Westlye ET, Fjaer S, Espeseth T, Lundervold A, Lundervold AJ (2013) General fluid-type intelligence is related to indices of white matter structure in middle-aged and old adults. Neuroimage 83C:372-383.

Hayes AF (2009) Beyond Baron and Kenny: statistical mediation analysis in the new millennium. Commun Monogr 76:408-420.

He J, Wong VS, Fletcher E, Maillard P, Lee DY, losif AM, Singh B, Martinez O, Roach AE, Lockhart SN, Beckett L, Mungas D, Farias ST, Carmichael O, DeCarli C (2012) The contributions of MRIbased measures of gray matter, white matter hyperintensity, and white matter integrity to late-life cognition. AJNR Am J Neuroradiol 33:1797-1803.

Honey CJ, Sporns O, Cammoun L, Gigandet X, Thiran JP, Meuli R, Hagmann P (2009) Predicting human resting-state functional connectivity from structural connectivity. Proc Natl Acad Sci USA 106:2035-2040.

Jacobs HI, Leritz EC, Williams VJ, Van Boxtel MP, van der Elst W, Jolles J, Verhey FR, McGlinchey RE, Milberg WP, Salat DH (2013) Association between white matter microstructure, executive functions, and processing speed in older adults: the impact of vascular health. Hum Brain Mapp 34:77-95.

Johansen-Berg $H$, Behrens TE, editors. Diffusion MRI: from quantitative measurement to in vivo neuroanatomy. London: Academic Press.

Johnson MA, Diaz MT, Madden DJ (2013) Global versus tractspecific components of cerebral white matter integrity: relation to adult age and perceptual speed (submitted for publication).

Jones DK (2008) Studying connections in the living human brain with diffusion MRI. Cortex 44:936-952.

Jones DK, Knosche TR, Turner R (2013) White matter integrity, fiber count, and other fallacies: the do's and don'ts of diffusion MRI. Neuroimage 73:239-254.

Koenig KA, Sakaie KE, Lowe MJ, Lin J, Stone L, Bermel RA, Beall EB, Rao SM, Trapp BD, Phillips MD (2013) High spatial and angular resolution diffusion-weighted imaging reveals forniceal damage related to memory impairment. Magn Reson Imaging 31:695-699.

Kramer AF, Erickson KI (2007) Capitalizing on cortical plasticity: influence of physical activity on cognition and brain function. Trends Cogn Sci 11:342-348.

Le Bihan D (2003) Looking in the functional architecture of the brain with diffusion MRI. Nat Rev Neurosci 4:469-480.

Li YO, Yang FG, Nguyen CT, Cooper SR, LaHue SC, Venugopal S, Mukherjee P (2012) Independent component analysis of DTI reveals multivariate microstructural correlations of white matter in the human brain. Hum Brain Mapp 33:1431-1451.

Lockhart SN, Mayda AB, Roach AE, Fletcher E, Carmichael O, Maillard P, Schwarz CG, Yonelinas AP, Ranganath C, Decarli C (2012) Episodic memory function is associated with multiple measures of white matter integrity in cognitive aging. Front Hum Neurosci 6:56.

Lovden M, Laukka EJ, Rieckmann A, Kalpouzos G, Li TQ, Jonsson T, Wahlund LO, Fratiglioni L, Backman L (2013) The dimensionality of between-person differences in white matter microstructure in old age. Hum Brain Mapp 34:1386-1398.

Lustig C, Shah P, Seidler R, Reuter-Lorenz PA (2009) Aging, training, and the brain: a review and future directions. Neuropsychol Rev 19:504-522.

Mackey AP, Whitaker KJ, Bunge SA (2012) Experience-dependent plasticity in white matter microstructure: reasoning training alters structural connectivity. Front Neuroanat 6. http://dx.doi.org/ 10.3389/fnana.2012.00032.

Madden DJ (2001) Speed and timing of behavioral processes. In: Birren JE, Schaie KW, editors. Handbook of the psychology of aging. San Diego, CA: Academic Press. p. 288-312. 
Madden DJ, Bennett IJ, Song AW (2009a) Cerebral white matter integrity and cognitive aging: contributions from diffusion tensor imaging. Neuropsychol Rev 19:415-435.

Madden DJ, Whiting WL, Huettel SA, White LE, MacFall JR, Provenzale JM (2004) Diffusion tensor imaging of adult age differences in cerebral white matter: relation to response time. Neuroimage 21:1174-1181.

Madden DJ, Bennett IJ, Burzynska A, Potter GG, Chen NK, Song AW (2012) Diffusion tensor imaging of cerebral white matter integrity in cognitive aging. Biochim Biophys Acta 1822:386-400.

Madden DJ, Spaniol J, Whiting WL, Bucur B, Provenzale JM, Cabeza R, White LE, Huettel SA (2007) Adult age differences in the functional neuroanatomy of visual attention: a combined fMRI and DTI study. Neurobiol Aging 28:459-476.

Madden DJ, Costello MC, Dennis NA, Davis SW, Shepler AM, Spaniol J, Bucur B, Cabeza R (2010) Adult age differences in functional connectivity during executive control. Neuroimage 52:643-657.

Madden DJ, Spaniol J, Costello MC, Bucur B, White LE, Cabeza R, Davis SW, Dennis NA, Provenzale JM, Huettel SA (2009b) Cerebral white matter integrity mediates adult age differences in cognitive performance. J Cogn Neurosci 21:289-302.

Maillard P, Carmichael O, Fletcher E, Reed B, Mungas D, DeCarli C (2012) Coevolution of white matter hyperintensities and cognition in the elderly. Neurology 79:442-448.

Maillard P, Fletcher E, Harvey D, Carmichael O, Reed B, Mungas D, DeCarli C (2011) White matter hyperintensity penumbra. Stroke 42:1917-1922.

Malloy P, Correia S, Stebbins G, Laidlaw DH (2007) Neuroimaging of white matter in aging and dementia. Clin Neuropsych 21: 73-109.

Marner L, Nyengaard JR, Tang Y, Pakkenberg B (2003) Marked loss of myelinated nerve fibers in the human brain with age. J Comp Neurol 462:144-152.

McIntosh AR (2000) Towards a network theory of cognition. Neural Netw 13:861-870.

Meier-Ruge W, Ulrich J, Bruhlmann M, Meier E (1992) Age-related white matter atrophy in the human brain. Ann NY Acad Sci 673:260-269.

Meier IB, Manly JJ, Provenzano FA, Louie KS, Wasserman BT, Griffith EY, Hector JT, Allocco E, Brickman AM (2012) White matter predictors of cognitive functioning in older adults. J Int Neuropsychol Soc 18:414-427.

Mesulam MM (1990) Large-scale neurocognitive networks and distributed processing for attention, language, and memory. Ann Neurol 28:597-613.

Metzler-Baddeley C, Jones DK, Belaroussi B, Aggleton JP, O'Sullivan MJ (2011) Frontotemporal connections in episodic memory and aging: a diffusion MRI tractography study. J Neurosci 31:13236-13245.

Michielse S, Coupland N, Camicioli R, Carter R, Seres P, Sabino J, Malykhin N (2010) Selective effects of aging on brain white matter microstructure: a diffusion tensor imaging tractography study. Neuroimage 52:1190-1201.

Minati L, Grisoli M, Bruzzone MG (2007) MR spectroscopy, functional $\mathrm{MRI}$, and diffusion-tensor imaging in the aging brain: a conceptual review. J Geriatr Psychiatry Neurol 20:3-21.

Mori S, van Zijl PC (2002) Fiber tracking: principles and strategies - a technical review. NMR Biomed 15:468-480.

Mungas D, Reed BR, Farias ST, Decarli C (2009) Age and education effects on relationships of cognitive test scores with brain structure in demographically diverse older persons. Psychol Aging 24:116-128.

Nagy Z, Alexander DC, Thomas DL, Weiskopf N, Sereno MI (2013) Using high angular resolution diffusion imaging data to discriminate cortical regions. PLoS One 8:e63842.

Nucifora PG, Verma R, Lee SK, Melhem ER (2007) Diffusion-tensor MR imaging and tractography: exploring brain microstructure and connectivity. Radiology 245:367-384.

O'Sullivan M, Jones DK, Summers PE, Morris RG, Williams SC, Markus HS (2001) Evidence for cortical "disconnection" as a mechanism of age-related cognitive decline. Neurology 57:632-638.

Pachella RG (1974) The interpretation of reaction time in informationprocessing research. In: Kantowitz B, editor. Human information processing: tutorials in performance and cognition. Hillsdale, NJ: Erlbaum. p. 41-82.

Park DC, Reuter-Lorenz P (2009) The adaptive brain: aging and neurocognitive scaffolding. Annu Rev Psychol 60:173-196.

Parks CM, losif A-M, Farias S, Reed B, Mungas D, DeCarli C (2011) Executive function mediates effects of white matter hyperintensities on episodic memory. Neuropsychologia 49:2817-2824.

Parks EL, Madden DJ (2013) Brain connectivity and visual attention. Brain Connect 3:317-338.

Penke L, Munoz Maniega S, Houlihan LM, Murray C, Gow AJ, Clayden JD, Bastin ME, Wardlaw JM, Deary IJ (2010a) White matter integrity in the splenium of the corpus callosum is related to successful cognitive aging and partly mediates the protective effect of an ancestral polymorphism in ADRB2. Behav Genet 40:146-156.

Penke L, Munoz Maniega S, Murray C, Gow AJ, Hernandez MC, Clayden JD, Starr JM, Wardlaw JM, Bastin ME, Deary IJ (2010b) A general factor of brain white matter integrity predicts information processing speed in healthy older people. J Neurosci 30:7569-7574.

Perry ME, McDonald CR, Hagler Jr DJ, Gharapetian L, Kuperman JM, Koyama AK, Dale AM, McEvoy LK (2009) White matter tracts associated with set-shifting in healthy aging. Neuropsychologia 47:2835-2842.

Peters A (2002) The effects of normal aging on myelin and nerve fibers: a review. J Neurocytol 31:581-593.

Pfefferbaum A, Sullivan EV, Hedehus M, Lim KO, Adalsteinsson E, Moseley M (2000) Age-related decline in brain white matter anisotropy measured with spatially corrected echo-planar diffusion tensor imaging. Magn Reson Med 44:259-268.

Pierpaoli C, Basser PJ (1996) Toward a quantitative assessment of diffusion anisotropy. Magn Reson Med 36:893-906.

Pierpaoli C, Jezzard P, Basser PJ, Barnett A, Di Chiro G (1996) Diffusion tensor MR imaging of the human brain. Radiology 201:637-648.

Ramnani N, Behrens TE, Penny W, Matthews PM (2004) New approaches for exploring anatomical and functional connectivity in the human brain. Biol Psychiatry 56:613-619.

Raz N, Lindenberger U, Rodrigue KM, Kennedy KM, Head D, Williamson A, Dahle C, Gerstorf D, Acker JD (2005) Regional brain changes in aging healthy adults: general trends, individual differences and modifiers. Cereb Cortex 15:1676-1689.

Salami A, Eriksson J, Nilsson LG, Nyberg L (2012) Age-related white matter microstructural differences partly mediate age-related decline in processing speed but not cognition. Biochim Biophys Acta 1822:408-415.

Salat DH (2011) The declining infrastructure of the aging brain. Brain Connect 1:279-293.

Salat DH, Tuch DS, Greve DN, van der Kouwe AJ, Hevelone ND, Zaleta AK, Rosen BR, Fischl B, Corkin S, Rosas HD, Dale AM (2005) Age-related alterations in white matter microstructure measured by diffusion tensor imaging. Neurobiol Aging 26:1215-1227.

Salthouse TA (1985) Speed of behavior and its implications for cognition. In: Birren JE, Schaie KW, editors. Handbook of the psychology of aging. New York: Van Nostrand Reinhold. p. 400-426.

Salthouse TA (1992) Mechanisms of age-cognition relations in adulthood. Hillsdale, NJ: Erlbaum.

Salthouse TA (1996) The processing-speed theory of adult age differences in cognition. Psychol Rev 103:403-428.

Salthouse TA (2006) Mental exercise and mental aging: evaluating the validity of the "Use It or Lose It" hypothesis. Perspect Psychol Sci 1:68-87.

Salthouse TA (2011) Neuroanatomical substrates of age-related cognitive decline. Psychol Bull 137:753-784. 
Salthouse TA, Madden DJ (2007) Information processing speed and aging. In: Deluca J, Kalmar J, editors. Information processing speed in clinical populations. New York: Psychology Press. p. 221-241.

Samanez-Larkin GR, Levens SM, Perry LM, Dougherty RF, Knutson B (2012) Frontostriatal white matter integrity mediates adult age differences in probabilistic reward learning. J Neurosci 32:5333-5337.

Sasson E, Doniger GM, Pasternak O, Tarrasch R, Assaf Y (2012) Structural correlates of cognitive domains in normal aging with diffusion tensor imaging. Brain Struct Funct 217:503-515.

Sasson E, Doniger GM, Pasternak O, Tarrasch R, Assaf Y (2013) White matter correlates of cognitive domains in normal aging with diffusion tensor imaging. Front Neurosci 7:32.

Schiavone F, Charlton RA, Barrick TR, Morris RG, Markus HS (2009) Imaging age-related cognitive decline: a comparison of diffusion tensor and magnetization transfer MRI. J Magn Reson Imaging 29:23-30.

Schneider BA, Pichora-Fuller MK (2000) Implication of perceptual deterioration for cognitive aging research. In: Craik FIM, Salthouse TA, editors. The handbook of aging and cognition. Mahwah, NJ: Erlbaum. p. 155-219.

Schulze ET, Geary EK, Susmaras TM, Paliga JT, Maki PM, Little DM (2011) Anatomical correlates of age-related working memory declines. J Aging Res 2011:606871.

Sexton CE, Mackay CE, Lonie JA, Bastin ME, Terriere E, O'Carroll RE, Ebmeier KP (2010) MRI correlates of episodic memory in Alzheimer's disease, mild cognitive impairment, and healthy aging. Psychiatry Res 184:57-62.

Smith EE, Salat DH, Jeng J, McCreary CR, Fischl B, Schmahmann JD, Dickerson BC, Viswanathan A, Albert MS, Blacker D, Greenberg SM (2011) Correlations between MRI white matter lesion location and executive function and episodic memory. Neurology 76:1492-1499.

Smith SM, Jenkinson M, Johansen-Berg H, Rueckert D, Nichols TE, Mackay CE, Watkins KE, Ciccarelli O, Cader MZ, Matthews PM, Behrens TE (2006) Tract-based spatial statistics: voxelwise analysis of multi-subject diffusion data. Neuroimage 31:1487-1505.

Song SK, Sun SW, Ju WK, Lin SJ, Cross AH, Neufeld AH (2003) Diffusion tensor imaging detects and differentiates axon and myelin degeneration in mouse optic nerve after retinal ischemia. Neuroimage 20:1714-1722.

Song SK, Yoshino J, Le TQ, Lin SJ, Sun SW, Cross AH, Armstrong RC (2005) Demyelination increases radial diffusivity in corpus callosum of mouse brain. Neuroimage 26:132-140.

Stadlbauer A, Salomonowitz E, Strunk G, Hammen T, Ganslandt O (2008) Quantitative diffusion tensor fiber tracking of age-related changes in the limbic system. Eur Radiol 18:130-137.

Stamatakis EA, Shafto MA, Williams G, Tam P, Tyler LK (2011) White matter changes and word finding failures with increasing age. PLoS One 6:e14496.

Sullivan EV, Pfefferbaum A (2003) Diffusion tensor imaging in normal aging and neuropsychiatric disorders. Eur J Radiol 45:244-255.

Sullivan EV, Pfefferbaum A (2006) Diffusion tensor imaging and aging. Neurosci Biobehav Rev 30:749-761.

Sullivan EV, Pfefferbaum A (2007) Neuroradiological characterization of normal adult ageing. $\mathrm{Br} J$ Radiol 80 (Spec No. 2): S99-S108.

Sullivan EV, Rohlfing T, Pfefferbaum A (2010a) Quantitative fiber tracking of lateral and interhemispheric white matter systems in normal aging: relations to timed performance. Neurobiol Aging 31:464-481.

Sullivan EV, Zahr NM, Rohlfing T, Pfefferbaum A (2010b) Fiber tracking functionally distinct components of the internal capsule. Neuropsychologia 48:4155-4163.

Sullivan EV, Adalsteinsson E, Hedehus M, Ju C, Moseley M, Lim KO, Pfefferbaum A (2001) Equivalent disruption of regional white matter microstructure in ageing healthy men and women. Neuroreport 12:99-104.
Sun SW, Liang HF, Cross AH, Song SK (2008) Evolving Wallerian degeneration after transient retinal ischemia in mice characterized by diffusion tensor imaging. Neuroimage 40:1-10.

Sun SW, Liang HF, Le TQ, Armstrong RC, Cross AH, Song SK (2006) Differential sensitivity of in vivo and ex vivo diffusion tensor imaging to evolving optic nerve injury in mice with retinal ischemia. Neuroimage 32:1195-1204.

Tamnes CK, Fjell AM, Østby Y, Westlye LT, Due-Tønnessen P, Bjørnerud A, Walhovd KB (2011) The brain dynamics of intellectual development: waxing and waning white and gray matter. Neuropsychologia 49:3605-3611.

Tang Y, Nyengaard JR, Pakkenberg B, Gundersen HJ (1997) Ageinduced white matter changes in the human brain: a stereological investigation. Neurobiol Aging 18:609-615.

Teipel SJ, Bokde AL, Meindl T, Amaro Jr E, Soldner J, Reiser MF, Herpertz SC, Moller HJ, Hampel H (2010) White matter microstructure underlying default mode network connectivity in the human brain. Neuroimage 49:2021-2032.

Tuch DS, Reese TG, Wiegell MR, Van JW (2003) Diffusion MRI of complex neural architecture. Neuron 40:885-895.

Valdes Hernandez MD, Booth T, Murray C, Gow AJ, Penke L, Morris Z, Maniega SM, Royle NA, Aribisala BS, Bastin ME, Starr JM, Deary IJ, Wardlaw JM (2013) Brain white matter damage in aging and cognitive ability in youth and older age. Neurobiol Aging [Epub ahead of print].

Van Impe A, Coxon JP, Goble DJ, Doumas M, Swinnen SP (2012) White matter fractional anisotropy predicts balance performance in older adults. Neurobiol Aging 33:1900-1912.

Vernooij MW, de Groot M, van der Lugt A, Ikram MA, Krestin GP, Hofman A, Niessen WJ, Breteler MM (2008) White matter atrophy and lesion formation explain the loss of structural integrity of white matter in aging. Neuroimage 43:470-477.

Vernooij MW, Ikram MA, Vrooman HA, Wielopolski PA, Krestin GP, Hofman A, Niessen WJ, Van der Lugt A, Breteler MM (2009) White matter microstructural integrity and cognitive function in a general elderly population. Arch Gen Psychiatry 66:545-553.

Wakana S, Jiang H, Nagae-Poetscher LM, van Zijl PC, Mori S (2004) Fiber tract-based atlas of human white matter anatomy. Radiology 230:77-87.

Westlye LT, Walhovd KB, Dale AM, Bjornerud A, Due-Tonnessen P, Engvig A, Grydeland H, Tamnes CK, Ostby Y, Fjell AM (2010) Life-span changes of the human brain white matter: diffusion tensor imaging (DTI) and volumetry. Cereb Cortex 20:2055-2068.

Wheeler-Kingshott CA, Cercignani M (2009) About "axial" and "radial" diffusivities. Magn Reson Med 61:1255-1260.

Wozniak JR, Lim KO (2006) Advances in white matter imaging: a review of in vivo magnetic resonance methodologies and their applicability to the study of development and aging. Neurosci Biobehav Rev 30:762-774.

Yassa MA, Muftuler LT, Stark CE (2010) Ultrahigh-resolution microstructural diffusion tensor imaging reveals perforant path degradation in aged humans in vivo. Proc Natl Acad Sci USA 107:12687-12691.

Yeo BT, Krienen FM, Sepulcre J, Sabuncu MR, Lashkari D, Hollinshead M, Roffman JL, Smoller JW, Zollei L, Polimeni JR, Fischl B, Liu H, Buckner RL (2011) The organization of the human cerebral cortex estimated by intrinsic functional connectivity. J Neurophysiol 106:1125-1165.

Young VG, Halliday GM, Kril JJ (2008) Neuropathologic correlates of white matter hyperintensities. Neurology 71:804-811.

Ystad M, Hodneland E, Adolfsdottir S, Haasz J, Lundervold AJ, Eichele T, Lundervold A (2011) Cortico-striatal connectivity and cognition in normal aging: a combined DTI and resting state fMRI study. Neuroimage 55:24-31.

Zahr NM, Rohlfing T, Pfefferbaum A, Sullivan EV (2009) Problem solving, working memory, and motor correlates of association and commissural fiber bundles in normal aging: a quantitative fiber tracking study. Neuroimage 44:1050-1062.

Zhan L, Jahanshad N, Ennis DB, Jin Y, Bernstein MA, Borowski BJ, Jack Jr CR, Toga AW, Leow AD, Thompson PM (2013) Angular 
versus spatial resolution trade-offs for diffusion imaging under time constraints. Hum Brain Mapp 34:2688-2706.

Zhang Y, Du AT, Hayasaka S, Jahng GH, Hlavin J, Zhan W, Weiner MW, Schuff N (2010) Patterns of age-related water diffusion changes in human brain by concordance and discordance analysis. Neurobiol Aging 31:1991-2001.

Zhu Z, Johnson NF, Kim C, Gold BT (2013) Reduced frontal cortex efficiency is associated with lower white matter integrity in aging. Cereb Cortex [Epub ahead of print]. 